\title{
Peroxiredoxin 4 Protects Against Nonalcoholic Steatohepatitis and Type 2 Diabetes in a Nongenetic Mouse Model
}

\author{
Atsunori Nabeshima,, ${ }^{1, *}$ Sohsuke Yamada, ${ }^{1, *}$ Xin Guo, Akihide Tanimoto,,2 Ke-Yong Wang, \\ Shohei Shimajiri, Satoshi Kimura, Takashi Tasaki, Hirotsugu Noguchi, Shohei Kitada,,3 \\ Teruo Watanabe, ${ }^{4}$ Junichi Fujii, ${ }^{5}$ Kimitoshi Kohno, ${ }^{6}$ and Yasuyuki Sasaguri ${ }^{1}$
}

\begin{abstract}
Aims: Consumption of a high-fructose diet (HFrD) can induce the development of a metabolic syndrome, manifesting as nonalcoholic steatohepatitis (NASH) and/or type 2 diabetes mellitus (T2DM), via a process in which oxidative stress plays a critical role. Peroxiredoxin 4 (PRDX4) is a unique and only known secretory member of the PRDX antioxidant family. However, its putative roles in the development of NASH and/or T2DM have not been investigated. Results: To elucidate the functions of PRDX4 in a metabolic syndrome, we established a nongenetic mouse model of T2DM by feeding mice a HFrD after injecting a relatively low dose of streptozotocin. Compared with wild-type (WT), human PRDX4 transgenic (Tg) mice exhibited significant improvements in insulin resistance, characterized by a lower glucose and insulin concentration and faster responses in glucose tolerance tests. The liver of $\mathrm{Tg}$ also showed less severe vesicular steatosis, inflammation, and fibrosis, along with lower lipid concentrations, lower levels of oxidative stress markers, more decreased expression of hepatic aminotransferase, and more reduced stellate cell activation than those in the WT liver, reminiscent of human early NASH. Hepatocyte apoptosis was also significantly repressed in Tg mice. By contrast, serum adiponectin levels and hepatic adiponectin receptor expression were significantly lower in WT mice, consistent with greater insulin resistance in the peripheral liver tissue compared with $\mathrm{Tg}$ mice. Innovation and Conclusion: Our data for the first time show that PRDX4 may protect against NASH, T2DM, and the metabolic syndrome by ameliorating oxidative stress-induced injury. Antioxid. Redox Signal. 19, 1983-1998.
\end{abstract}

\section{Introduction}

$\mathbf{N}$ ONALCOHOLIC FATTY LIVER DISEASE (NAFLD) is one of the most common forms of liver disease in children and adults, and is estimated to affect about $20 \%-30 \%$ of people especially in developed countries $(2,14)$. NAFLD, defined as the accumulation of triglycerides (TG) in individuals who do not consume a large amount of alcohol $(<20 \mathrm{~g}$ ethanol/day), is a clinicopathological syndrome with wide-spectrum presenta- tions, ranging from hepatic simple steatosis to nonalcoholic steatohepatitis (NASH) and cirrhosis $(14,19,23)$. NASH is a more severe form of NAFLD, and usually progresses to end-stage liver diseases, including cirrhosis and/or hepatocellular carcinoma $(14,23)$. NASH is also closely associated with obesity, insulin resistance, and metabolic syndrome, and the liver is recognized as one of the pathological targets of metabolic syndrome $(1,23)$.

The current increase in the prevalence of NAFLD may reflect changes in the environmental factors, particularly, diet

\footnotetext{
${ }^{1}$ Department of Pathology and Cell Biology, School of Medicine, University of Occupational and Environmental Health, Kitakyushu, Japan.

${ }^{2}$ Department of Tumor Pathology, Field of Oncology, Kagoshima University Graduate School of Medical and Dental Sciences, Kagoshima, Japan.

${ }^{3}$ Department of Urology, School of Medicine, University of Occupational and Environmental Health, Kitakyushu, Japan.

${ }^{4}$ Laboratory of Pathology, Fukuoka Wajiro Hospital, Fukuoka, Japan.

${ }^{5}$ Department of Biomolecular Function, Graduate School of Medical Science, Yamagata University, Yamagata, Japan.

${ }^{6}$ Department of Molecular Biology, School of Medicine, University of Occupational and Environmental Health, Kitakyushu, Japan.

*These two authors contributed equally to this study.
} 


\section{Innovation}

No prior studies have investigated the potential roles of peroxiredoxin 4 (PRDX4) in the development of nonalcoholic fatty liver disease (NAFLD) and/or type 2 diabetes mellitus (T2DM). Here we show for the first time that PRDX4 protects against the progression of nonalcoholic steatohepatitis and insulin resistance, particularly in the liver, in a nongenetic mouse model of NAFLD/T2DM. These effects of PRDX4 were achieved by reducing oxidative stress and ameliorating hepatic steatosis, inflammatory reaction, apoptotic activity, and fibrogenesis at systemic as well as local levels. Our present findings, together with those of our previous studies, provide novel insights into the protective role of PRDX4 in the development of the metabolic syndrome, manifesting as obesity, T2DM, NAFLD, and/or atherosclerosis.

and physical activity, paralleling the obesity epidemic $(2,14$, $19,23)$. Along with an increase in total energy consumption, there has been a substantial increase in dietary fructose consumption caused by a high intake of sucrose and highfructose corn syrup, common sugar-sweeteners used in the food industry $(8,26)$. In fact, high fructose consumption is closely associated with childhood obesity and resulted in the occurrence of type 2 diabetes mellitus (T2DM) in adults (16, 22). In rodent models, consumption of a high-fructose diet (HFrD) almost invariably induces the concomitant development of metabolic syndrome, characterized by excess body fat, insulin resistance, hypertriglyceridemia, and/or NASH $(12,13,26)$. Oxidative stress and endoplasmic reticulum (ER) stress appear to be involved in these processes $(3,21,28)$. Luo et al. previously described that the metabolic characteristics of a nongenetic mouse model, in which C57BL/ 6 mice were fed a HFrD for more than 7 weeks after an intraperitoneal injection of low-dose streptozotocin (STZ), were similar to those of T2DM patients, and that its model did not impose heavy burdens, such as availability or cost, on researchers (17). According to the two-hit hypothesis of NASH, the second hits include cytokine, metabolic, and oxidative stressors that overwhelm hepatocyte endogenous survival mechanisms. These hits promote hepatocyte apoptosis and regenerative mechanisms and subsequently drive the progression from simple steatosis to NASH and cirrhosis $(4,25)$. However, very few reports of experimental animals fed a HFrD described the histopathological features of the liver. Additionally, to our knowledge, no studies have examined the close relationship between oxidative stress and NASH and/or T2DM in a nongenetic mouse model.

A recently discovered family of antioxidant proteins, designated peroxiredoxin (PRDX), is ubiquitously synthesized and abundantly expresses in various organisms (24). The PRDX family includes at least six distinct PRDX genes expressed in mammals. Its functional peroxidase activity is dependent on reduced forms of thioredoxin and/or glutathione $(11,24)$. Human PRDX4 (hPRDX4) was first identified in a yeast two hybrid screening assay as a partner for a thiolspecific antioxidant (6). Unlike the intracellular localization of other family members, PRDX4 is the only known secretory form $(6,10)$, and protects against oxidative damage by scavenging reactive oxygen species (ROS) in the extracellular space $(10,18)$. We previously generated $h P R D X 4$ transgenic (Tg) mice from a C57BL/ 6 background and evaluated the role of PRDX 4 in a model of type 1 diabetes mellitus (T1DM). We found that PRDX4-expressing $\beta$-cells in the islets of Tg mice were significantly protected against inflammation and apoptosis, unlike those of control C57BL/ 6 wild-type (WT) mice $(5,29)$. More recently, it was found that PRDX4 also ameliorated the progression of atherosclerosis in apolipoprotein E-knockout mice fed a high-cholesterol diet by protecting the aorta from oxidative stress and reducing apoptosis of the endothelium, neointimal macrophages, and smooth muscle cells (7). By contrast, the pathophysiological relevance of the PRDX family, including PRDX4, in NASH and/or T2DM remains to be clarified.

In this study, we for the first time investigated the roles of PRDX4 in the pathogenic mechanisms underlying T2DM and NAFLD, especially NASH. Inducing this nongenetic mouse model in Tg and WT mice has allowed us to obtain novel insights into the protective function of PRDX4 on the development of T2DM and NASH. The results suggest that PRDX4 might help to protect against the metabolic syndrome by reducing oxidative stress and suppressing steatosis, inflammatory reactions, and apoptotic activity in the liver.

\section{Results}

\section{Expression of PRDX4 in mice under basal conditions and after inducing the nongenetic mouse model}

The mRNA and protein expression levels of PRDX4 in various tissues from nontreated $\mathrm{Tg}$ mice were detected by reverse transcriptase (RT-)- and real-time polymerase chain reaction (PCR) and Western blotting, respectively $(5,7,29)$. PCR clearly revealed the expression of endogenous mouse PRDX4 (mPRDX4) in the liver of $\mathrm{Tg}$ mice with or without induction of the nongenetic mouse model (Fig. 1A). However, $h P R D X 4$ was only detected in Tg mice, and its expression was significantly higher compared with mPRDX4 (Fig. 1A, B). Additionally, its expression was significantly greater than in $\mathrm{Tg}$ mice on the nongenetic mouse model than in noninduced Tg mice (Fig. 1A). Real-time RT-PCR showed that the gene expression levels of most of the $m P R D X s$ examined were not significantly different between the two groups of the nongenetic mouse model, even though that of endogenous mPRDX4 was significantly upregulated in Tg mice (Fig. 1C). On the other hand, immunofluorescence studies showed that hepatocytes obtained from Tg mice, but not those from WT mice, expressed intracellular hPRDX4, colocalized with ER-Tracker Blue-White (Fig. 2A). However, morphological and biochemical studies revealed no difference in gross appearances of the liver, whole-body glucose homeostasis, or insulin resistance under basal conditions between $\mathrm{Tg}$ and WT mice (data not shown) (5).

In mice subjected to the nongenetic mouse model (HFrD and low-dose STZ injection), immunohistochemistry (IHC) (Fig. 2B) and Western blotting (Fig. 2C) confirmed that the hPRDX4 protein was highly expressed throughout the liver of $\mathrm{Tg}$ mice. To determine the circulatory level of the hPRDX4 protein, we performed enzyme-linked immunosorbent assays (ELISA) using serum samples obtained after inducing the nongenetic mouse model. These tests confirmed that Tg mice had significantly elevated PRDX4 levels compared with WT 

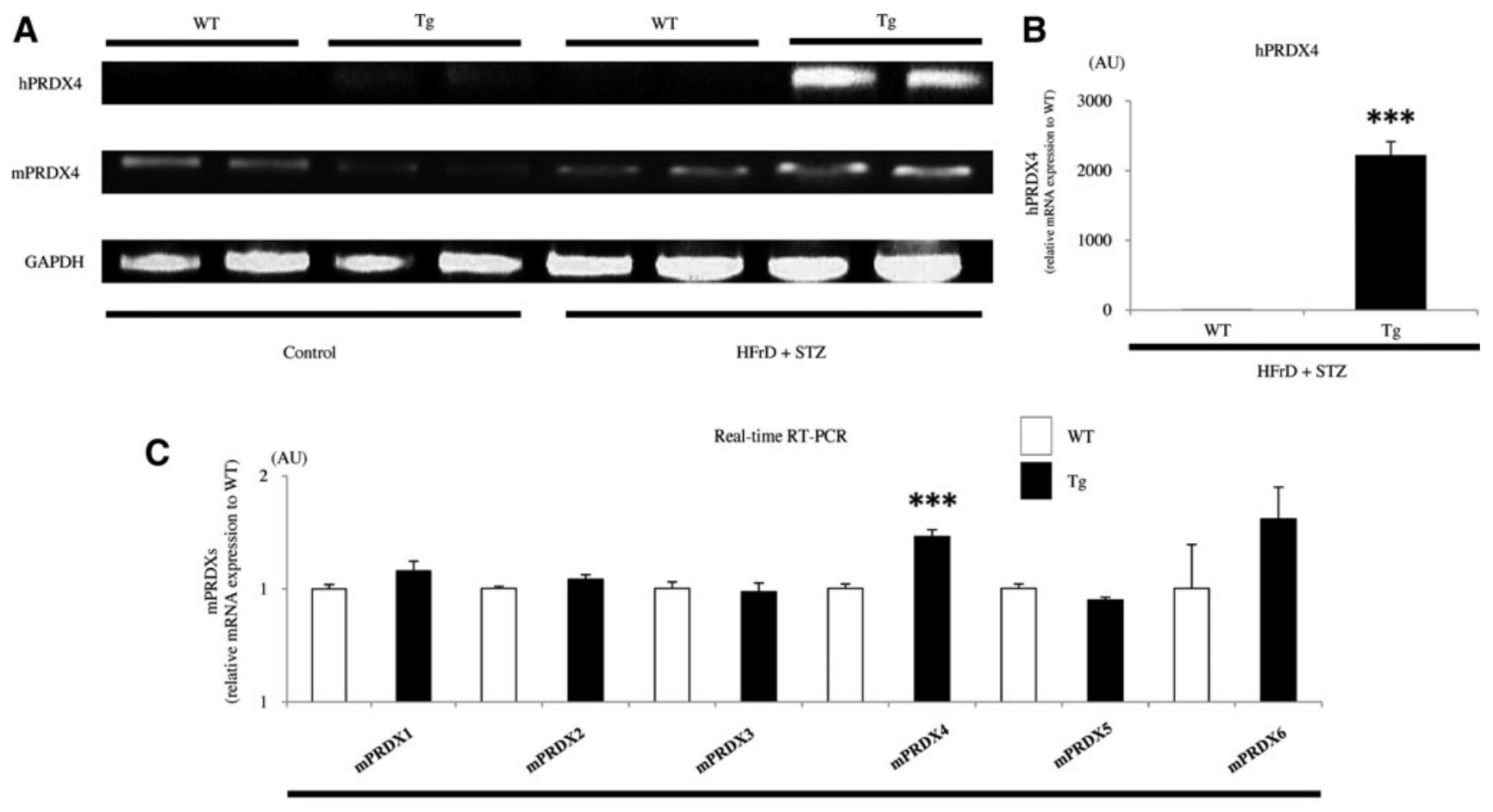

HFrD + STZ

FIG. 1. Analysis of the expression of PRDX mRNA in the mice. (A) RT-PCR showed that the liver of WT and Tg mice expressed endogenous mPRDX4. WT mice did not express hPRDX4. The expression of hPRDX4 was significantly greater compared with mPRDX4 in both control ( $n=5$ mice per group) and HFrD + STZ ( $n=10$ mice per group) $\mathrm{Tg}$ mice. In Tg mice, hPRDX4 expression was also significantly greater in HFrD+STZ mice than in control mice. (B) Real-time RT-PCR confirmed that hPRDX4 expression was markedly increased in HFrD $+\mathrm{STZ}$ Tg mice $(n=10$ mice per group) $(* * * p<0.0001)$. (C) Expression levels of mPRDX1, 2, 3, 5, and 6 were not significantly different between WT and Tg mice $(\mathrm{HFrD}+\mathrm{STZ}, n=10$ mice per group). However, hepatic expression of endogenous mPRDX4 was significantly higher in Tg mice than in WT mice $\left({ }^{* * *} p<0.0001\right)$. Values are means $\pm S E$ in $B-C$ and were normalized for $18 S$ rRNA expression (real-time RT-PCR) or GAPDH expression (RT-PCR) in (A-C). HFrD, high-fructose diet; hPRDX4, human peroxiredoxin 4; mPRDX4, mouse peroxiredoxin 4; RT-PCR, reverse transcriptase-polymerase chain reaction; STZ, streptozotocin; Tg, hPRDX4 transgenic; WT, wild type; GAPDH, glyceraldehydes 3-phosphate dehydrogenase.

mice (WT $7.0 \pm 4.1 \mathrm{pg} / \mathrm{ml}$ vs. Tg $22.8 \pm 4.0 \mathrm{pg} / \mathrm{ml} ; p<0.05$ ) (Fig. 2D). Western blotting analysis (Fig. 2C) showed that hPRDX4 was significantly expressed in the liver of model $\mathrm{Tg}$ mice, whereas the liver of WT mice had a faint band corresponding to the anti-hPRDX4 antibody, indicating cross-reactivity of the antibodies against hPRDX4 to mPRDX4. IHC also confirmed that hPRDX4 was expressed in hepatocytes from model $\mathrm{Tg}$ mice, but not or was very weakly in hepatocytes from model WT mice (Fig. 2B).

\section{Comparison of metabolic parameters between WT and $\mathrm{Tg}$ mice after inducing the nongenetic mouse model or after being fed a HFrD alone}

Blood glucose was measured at 1-4 weeks after STZ injection. All STZ-injected mice developed hyperglycemia as a model of DM, but the increase in blood glucose from 3 to 4 weeks after STZ injection was slower and smaller in Tg mice than in WT mice $(p<0.05$; Fig. 3A). Serum insulin levels, as measured by ELISA, were not significantly different between WT and Tg mice before STZ injection (WT $1.07 \pm 0.02 \mathrm{ng} / \mathrm{ml}$ vs. $\operatorname{Tg} 1.19 \pm 0.09 \mathrm{ng} / \mathrm{ml})$. However, insulin levels at 4 weeks after STZ injection were significantly greater in WT than in $\mathrm{Tg}$ mice (WT $1.54 \pm 0.22 \mathrm{ng} / \mathrm{ml}$ vs. Tg $1.03 \pm 0.09 \mathrm{ng} / \mathrm{ml}$ ), as well as that of WT mice before STZ injection $(p<0.05$; Fig. 3B). We next performed the glucose tolerance tests, and found that the glucose levels reached a peak at $30 \mathrm{~min}$ after glucose injection (Fig. 3C). The normalization of blood glucose levels between 15 and $60 \mathrm{~min}$ after injection was much faster in $\mathrm{Tg}$ mice compared with WT mice ( $p<0.05$; Fig. 3C). Blood glucose levels measured before and after insulin injection tended to be lower and showed a faster response in Tg mice than in WT mice injected with STZ, but the differences were not statistically significant between the two groups from 15 to $120 \mathrm{~min}$ on the glucose response test (data not shown). These features apparently manifested as insulin resistance in the model WT mice. Additionally, the pancreatic islet area was significantly smaller in mice at 4 weeks after STZ injection than in untreated mice $(p<0.05$; Supplementary Fig. S1B; Supplementary Data are available online at www.liebertpub.com/ars), but was not significantly different between WT and $\mathrm{Tg}$ mice at either 0 weeks (WT $24.1 \pm 2.8 \times 10^{3} \mu \mathrm{m}^{2}$ vs. $\mathrm{Tg} 23.4 \pm 3.0 \times 10^{3} \mu \mathrm{m}^{2}$ ) or 4 weeks after injection (WT $10.2 \pm 1.7 \times 10^{3} \mu \mathrm{m}^{2}$ vs. Tg $9.6 \pm$ $2.3 \times 10^{3} \mu \mathrm{m}^{2}$ ). The body weight (BW) (Fig. 4A) and organs (liver or white adipose tissue [WAT]))/BW ratios (Fig. 4B) revealed that the model was not overtly obese and not significantly different between WT and Tg mice at 4 weeks after STZ injection. In addition, food consumption measured using metabolic cages was slightly elevated after STZ injection than before injection in both two groups of WT and Tg mice, but was similar in both 
A

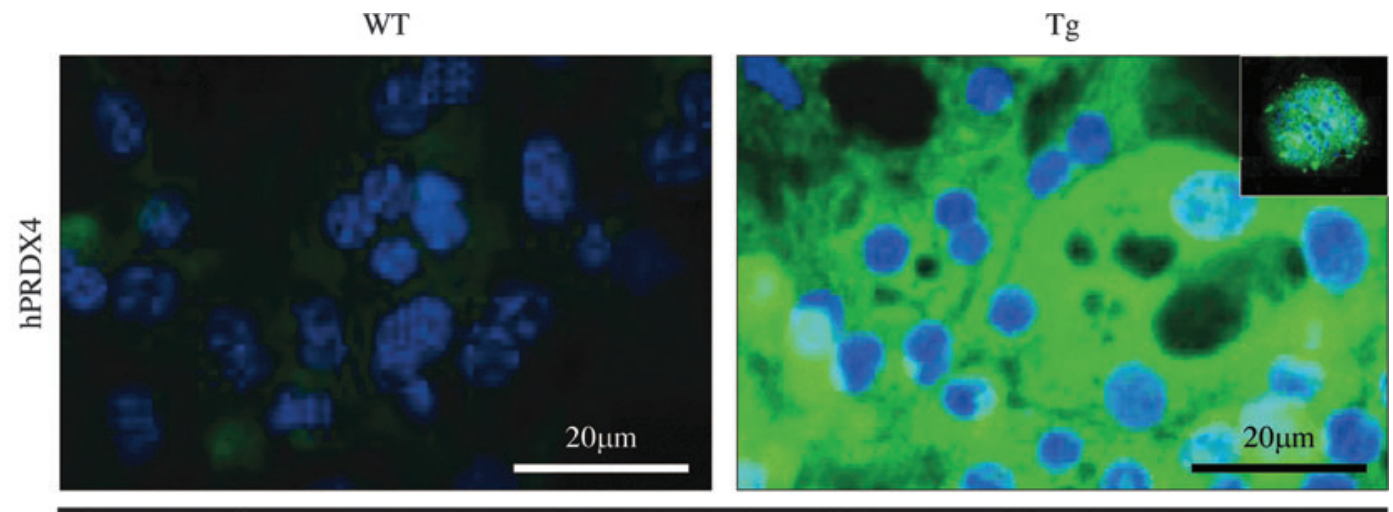

Control

B

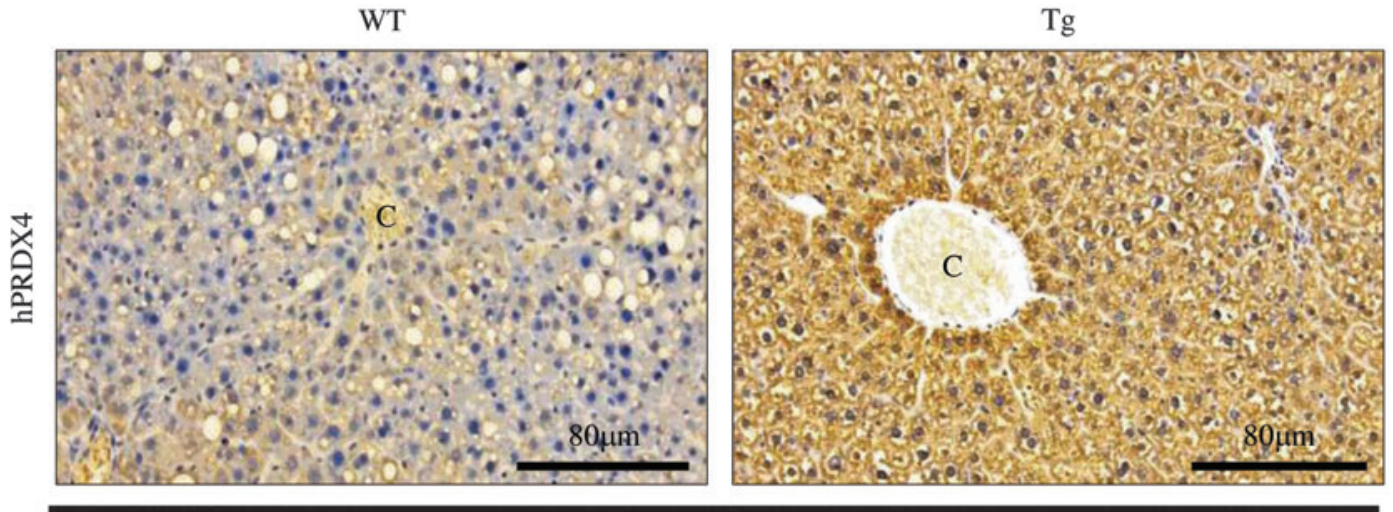

HFrD + STZ

C

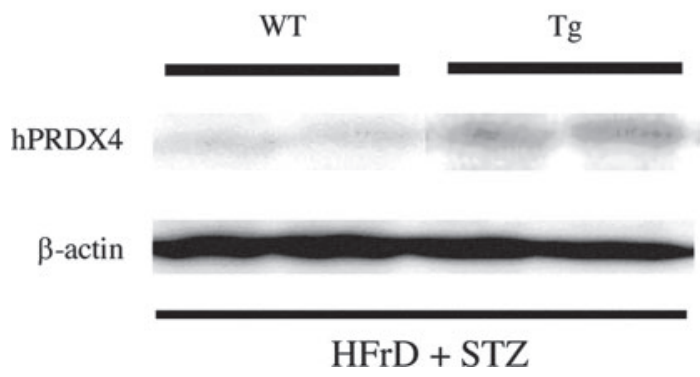

D

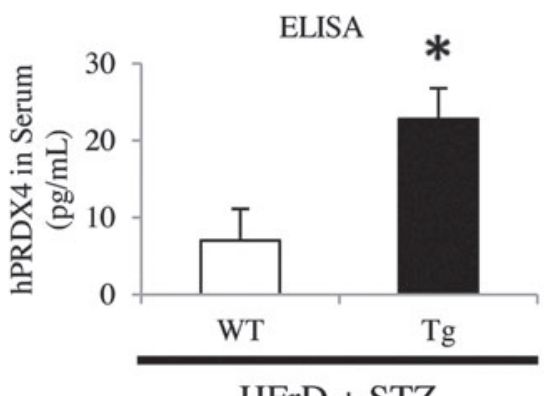

FIG. 2. Analysis of the expression of hPRDX4 protein in the mice. (A) Immunofluorescence staining showed that, in vitro, hPRDX4 was specifically expressed in hepatocytes harvested from Tg mice, but not in those from WT mice, under basal conditions (control, $n=5$ mice per group). The hPRDX4 protein and ER-Tracker Blue-White were intracellularly colocalized in hepatocytes obtained from Tg mice (inset). Scale bar $=20 \mu \mathrm{m}$. (B) At 8 weeks after HFrD feeding and STZ injection (HFrD +STZ, $n=10$ mice per group), hPRDX4 was detected in many hepatocytes throughout the liver of Tg mice, and was weakly detected in a very small number of hepatocytes from WT mice, indicating weak cross-reactivity between hPRDX4 and endogenous mPRDX4. C, central vein. Scale bar $=80 \mu \mathrm{m}$. (C) Western blotting showed increased hepatic hPRDX4 protein expression in HFrD + STZ Tg mice ( $n=10$ mice per group). Very faint bands were detected in WT mice, indicating weak cross-reactivity of the antibody for mPRDX4, as shown in Figure 2B. (D) ELISAs confirmed that serum hPRDX4 levels were elevated in $\mathrm{HFrD}+\mathrm{STZ} \mathrm{Tg}$ mice $(n=10 \mathrm{mice}$ per group), revealing that only Tg mice had markedly elevated hPRDX4 levels, but not in WT mice $\left({ }^{*} p<0.05\right)$. Values are means \pm SE and were normalized for $\beta$-actin expression (western blotting). ELISA, enzyme-linked immunosorbent assay; ER, endoplasmic reticulum. To see this illustration in color, the reader is referred to the web version of this article at www.liebertpub.com/ars

groups of mice (Fig. 4C). We also determined the serum cholesterol profiles by high-performance liquid chromatography (HPLC) in mice fed the HFrD for 8 weeks after STZ injection. Both WT and Tg mice developed modest hypolipidemia with mildly decreased levels of total cholesterol (T-cho), very lowdensity lipoprotein (VLDL), low-density lipoprotein (LDL), and
TG. However, the serum levels of T-cho, LDL, and high-density lipoprotein (HDL) were significantly higher in $\mathrm{Tg}$ mice than in WT mice ( $p<0.05$; Fig. 4D), while the serum TG level was significantly lower in Tg mice than in WT mice ( $p<0.05$; Fig. 4D). There were no significant differences in VLDL or free fatty acid (FFA) levels between the two groups (Fig. 4D). 

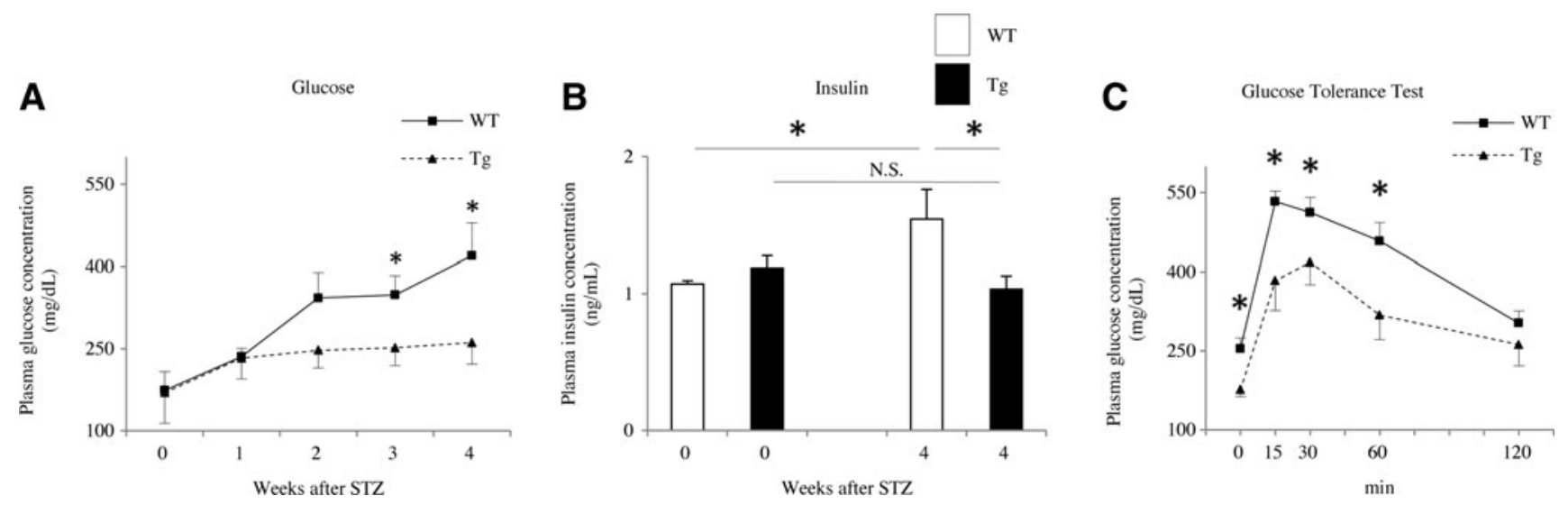

FIG. 3. Comparison of glucose metabolic parameters between WT and Tg mice following induction of the nongenetic mouse model. (A) Most of the mice developed hyperglycemia within 1 week after STZ injection, but the increase in blood glucose between weeks 1 and 4 was significantly slower and smaller in Tg mice than in WT mice $(\mathrm{HFrD}+\mathrm{STZ}, n=10 \mathrm{mice}$ per group). (B) Insulin concentrations, measured by ELISA, were not significantly different between WT and Tg mice before STZ injection. However, insulin levels were significantly higher in WT mice than in Tg mice after STZ injection $(\mathrm{HFrD}+\mathrm{STZ}, n=10$ mice per group). (C) During glucose tolerance tests, blood glucose concentrations increased within 15 min. However, normalization of blood glucose levels was significantly faster in Tg mice than in WT mice (HFrD $+\mathrm{STZ}, n=10$ mice per group). ${ }^{*} p<0.05$. Values are means $\pm \mathrm{SE}$.

Blood glucose levels did not increase significantly in either WT or Tg mice after being fed the HFrD alone for 20 weeks, and no significant differences were found between the two groups (Supplementary Fig. S2A). By contrast, the BW and the WAT/BW in Tg mice demonstrated a slower increase and a lower tendency for obesity compared with WT mice after 18-20 weeks of HFrD feeding $(p<0.05$; Supplementary Fig. $\mathrm{S} 2 \mathrm{~B}, \mathrm{C})$. Cell counting revealed that WAT from HFrD-fed Tg mice contained significantly more compared with HFrD-fed WT mice (WT 65.4 \pm 6.9 vs. Tg 135.0 \pm 8.3 ) ( $p<0.0001$; Supplementary Fig. S2D).

Blood pressure did not increase significantly in either WT or Tg mice after HFrD feeding with or without STZ injection. Furthermore, blood pressure was not significantly different between WT and Tg mice (data not shown).

\section{Histological and morphometric analyses of NAFLD in WT and Tg liver}

After 4 weeks of HFrD feeding and STZ injection, the liver tissues from $\mathrm{Tg}$ mice showed almost no changes in their gross appearance, whereas those from WT mice were pale and yellowish in color (Fig. 5A), indicative of lipid accumulation. Hematoxylin and eosin (H\&E) staining revealed that the $\mathrm{Tg}$ liver contained significantly fewer lipid droplets and scattered inflammatory foci (steatosis score: $0.38 \pm 0.26$; inflammation score: $0.50 \pm 0.25$ ), compared with those of WT mice (steatosis score: $2.38 \pm 0.14$; inflammation score: $1.25 \pm 0.15)(p<0.0001$ and $p<0.05$, respectively) (Fig. 5B-E). The lipid droplets in the NAFLD liver of WT mice were of varying size, indicating mixed microvesicular to macrovesicular steatosis (Fig. 5B, C). In addition, Oil Red-O staining confirmed greater hepatic lipid accumulation in WT mice (Fig. 5D), as the hepatic TG and FFA content was significantly greater in WT mice than in $\mathrm{Tg}$ mice (TG: WT $18.7 \pm 2.4 \mathrm{mg} / \mathrm{g}$ vs. Tg $11.5 \pm 1.6 \mathrm{mg} / \mathrm{g} ; p<0.05$ ) (FFA: WT $13.5 \pm 3.0 \mathrm{mEq} / \mathrm{g}$ vs. Tg $7.0 \pm 1.1 \mathrm{mEq} / \mathrm{g} ; p<0.05$ ) (Fig. 5F). Moreover, hepatocyte ballooning and anisonucleosis were occasionally seen particularly in the WT liver (Fig. 5C).
IHC for CD3 showed that the mild NAFLD liver in Tg mice contained fewer infiltrating $\mathrm{T}$ lymphocytes compared with that in WT mice (WT $9.3 \pm 1.1$ vs. Tg $3.7 \pm 1.0 ; p<0.05$ ) (Fig. $6 \mathrm{~A})$. Mac-2-staining also showed that there were fewer macrophages (Kupffer cells) per inflammatory foci in the Tg liver than in the WT liver (WT $42.1 \pm 1.5$ vs. Tg $34.4 \pm 2.7 ; p<0.05$ ) (Fig. 6B). HFrD feeding and STZ injection induced relatively progressive hepatic inflammation in WT mice. Additionally, fibrogenesis and stellate cell activation, as determined by picrosirius red staining and IHC for $\alpha$-smooth muscle actin $(\alpha-S M A)$, respectively, were not apparent in the $\mathrm{Tg}$ liver (Fig. 7A, B). In WT mice, picrosirius red staining revealed evidence of perivenular and pericellular fibrosis or fibrogenesis (WT $3.7 \% \pm 1.8 \%$ vs. Tg $0.10 \% \pm 0.05 \%$; $p<0.05$ ) (Fig. 7A), while IHC revealed specific and linear expression of $\alpha$-SMA along the hepatic sinusoids (WT $91.9 \pm 11.2$ vs. Tg 44.2 \pm 7.0 ; $p<0.05$ ) (Fig. 7B), consistent with activation of hepatic stellate cells in WT mice. Under polarized light, the red-stained fibers were identified as collagen type I because of predominant yellow birefringence (Fig. 7A). Furthermore, the serum level of alanine aminotransferase (ALT), but not of aspartate aminotransferase (AST), was significantly increased in WT mice, but not in Tg mice (Fig. 6C), indicative of hepatocyte cell death (apoptosis). These features, including the induction of macrovesicular steatosis by TG and FFA accumulation, ballooning of hepatocytes, inflammation, and fibrosis in the liver of WT mice, are similar to those seen in NASH in humans. In fact, the NASH score from the WT liver was significantly higher than that in the $\mathrm{Tg}$ liver after inducing the nongenetic mouse model (WT $2.60 \pm 0.54$ vs. $\operatorname{Tg} 1.00 \pm 0.49 ; p<0.05$ ) (Fig. 5E).

We also established a second model of NASH, in which Tg and WT mice were fed a methionine- and choline-deficient high-fat (MCD+HF) diet (15). Hepatic steatosis and inflammation were significantly reduced in $\mathrm{Tg}$ mice compared with those in WT mice (Supplementary Fig. S3A-C), consistent with the findings observed with HFrD feeding and STZ injection (nongenetic mouse model). Additionally, inflammation in the liver of $m P R D X 4$ knockout mice $\left(m P R D X 4^{-/ y}\right)(9)$ 

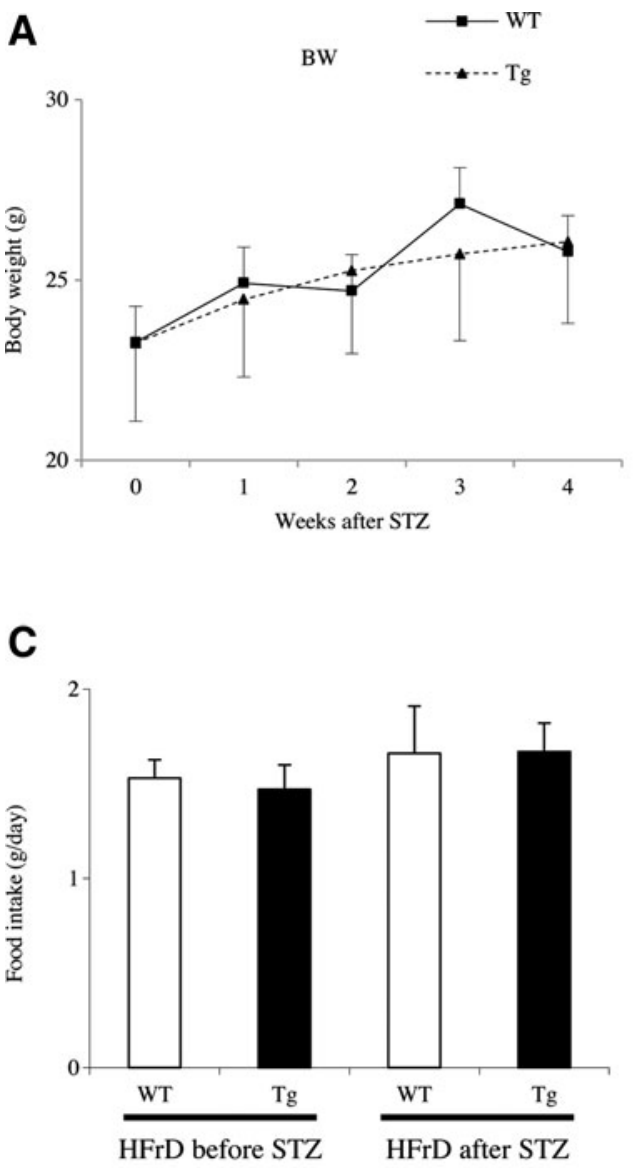

B
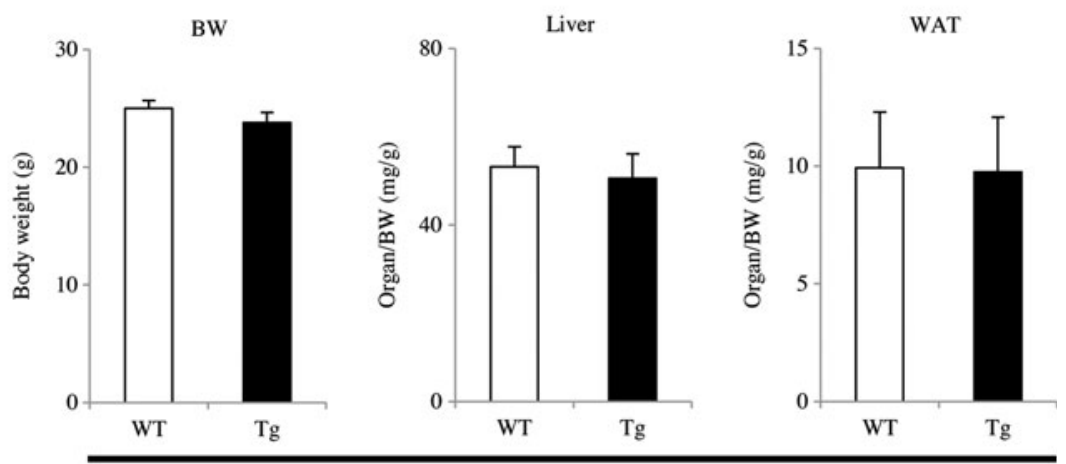

$\mathrm{HFrD}+\mathrm{STZ}$

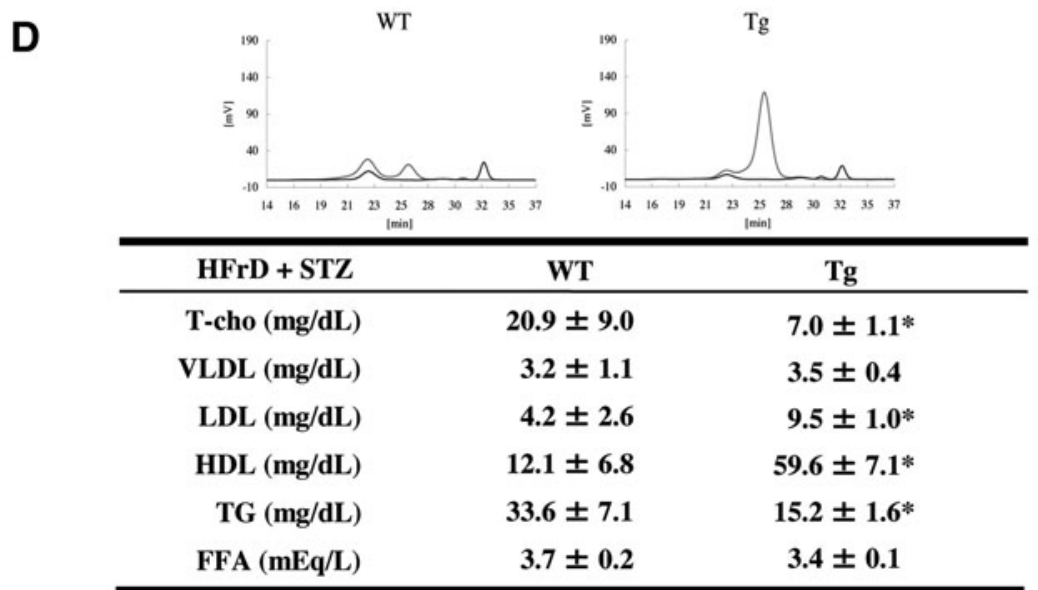

FIG. 4. Comparison of other metabolic parameters between WT and Tg mice following induction of the nongenetic mouse model. (A) BW was not significantly different between WT and Tg mice at weeks 0 and 4 of HFrD feeding and STZ injection (HFrD $+\mathrm{STZ}, n=10$ mice per group). Therefore, this nongenetic mouse model did not exhibit overt obesity. (B) BW and organ/BW ratios were not significantly different between Tg and WT mice at 4 weeks after STZ injection (HFrD+STZ, $n=10$ mice per group). (C) Although food consumption was slightly, but not significantly increased after STZ injection than before STZ injection in Tg and WT mice (HFrD $+\mathrm{STZ}, n=10$ mice per group), it was similar in Tg and WT mice at both times. (D) Serum cholesterol level. Upper, a representative serum cholesterol profile is shown, determined by HPLC in a mouse fed the HFrD for 8 weeks and injected with STZ injection (HFrD + STZ, $n=10$ mice per group). The concentration of lipoproteins is shown on the $y$ axis $(\mathrm{mV})$, and elution time is indicated on the $\mathrm{x}$ axis. Bottom, WT and Tg mice developed modest hypolipidemia with mild reductions of T-cho, VLDL, LDL, and TG. However, serum T-cho, LDL, and HDL levels were significantly higher and TG levels were significantly lower in Tg mice than in WT mice. There were no significant differences in VLDL or FFA levels between the two groups. ${ }^{*} p<0.05$. Values are means \pm SE. FFA, free fatty acid; LDL, low-density lipoprotein; VLDL, very low-density lipoprotein; TG, triglyceride; T-cho, total cholesterol; HDL, high-density lipoprotein.

was significantly more progressive than in the liver from WT mice, although the difference of steatosis was not significant (Supplementary Fig. S3A-C). Consequently, the NASH scores were significantly different between WT or mPRDX $4^{-/ y}$ mice and Tg mice (Supplementary Fig. S3D).

After 20 weeks of HFrD feeding without STZ injection, the livers from WT and Tg mice did not exhibit marked features of NAFLD and there were no significant differences between WT and Tg mice in terms of macroscopic or microscopic findings (Supplementary Fig. S2E).

\section{Hepatic expression of proinflammatory signaling factors in livers of WT and Tg mice after inducing the nongenetic mice model}

Real-time RT-PCR showed that the expression levels of proinflammatory signaling factors examined were not sig- nificantly different between WT and Tg mice. However, the expression levels of the tumor necrosis factor (TNF)- $\alpha$ and TNF receptor (TNFR) 2 were significantly downregulated in the livers of model Tg mice compared with those in model WT mice $(p<0.05)$ (Fig. 6D).

\section{Levels of 8-hydroxy-2 -deoxyguanosine and 4-hydroxy-2-nonenal in WT and Tg livers before and after inducing the nongenetic mouse model}

We used IHC to determine the expression of 8-hydroxy2'-deoxyguanosine (8-OHdG) and 4-hydroxy-2-nonenal (4-HNE) as markers for oxidative stress. The numbers of hepotocytes expressing both 8-OHdG- and 4-HNE were very low in untreated WT and Tg mice (data not shown), but their numbers were significantly increased by HFrD feeding and STZ injection. Nevertheless, there were significantly fewer 

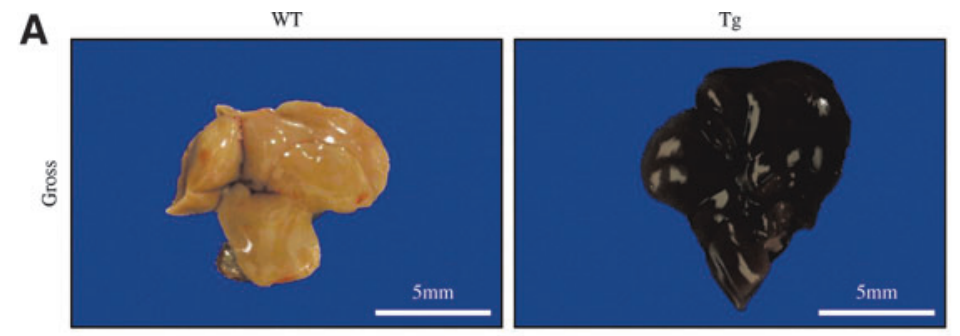

E
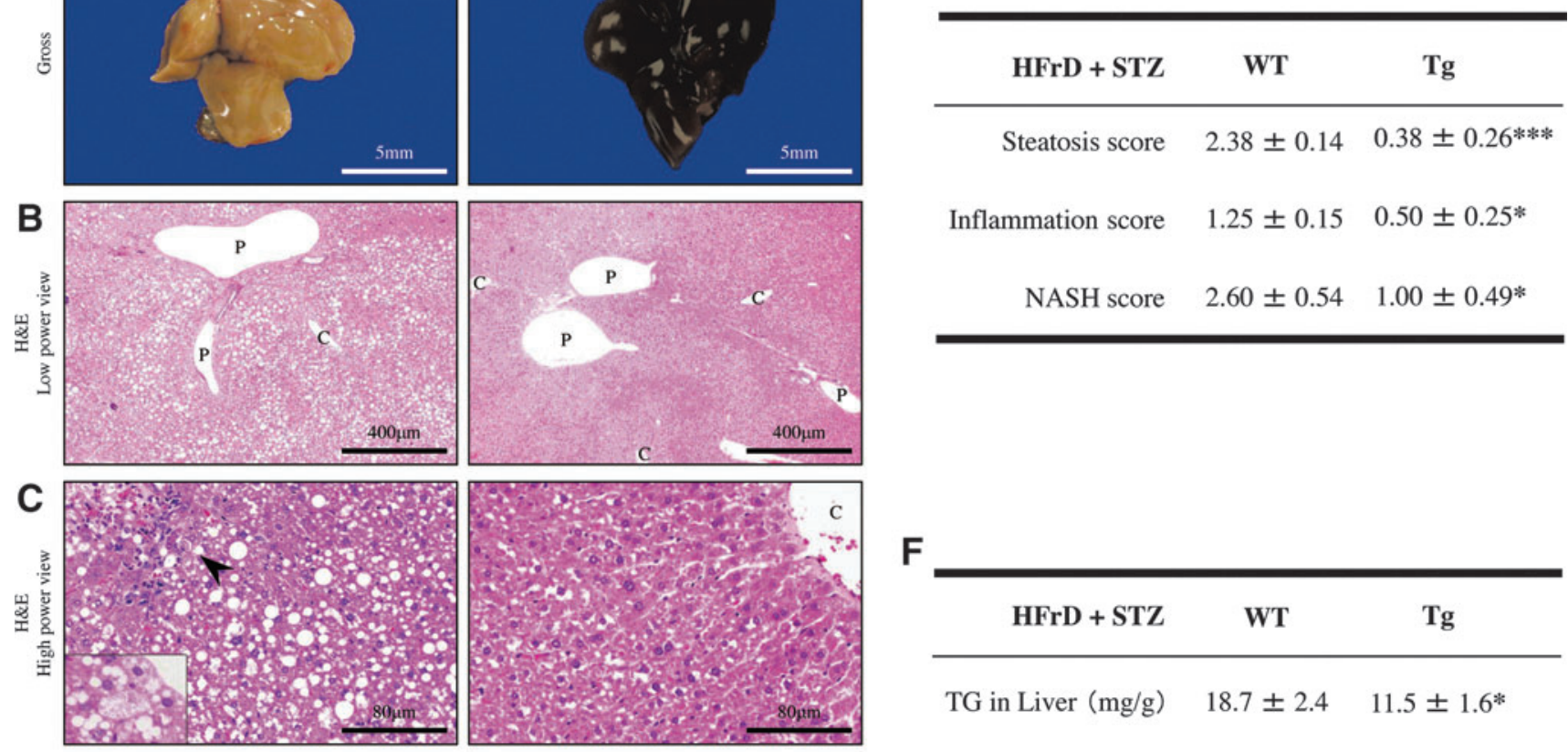

$\mathbf{F}$

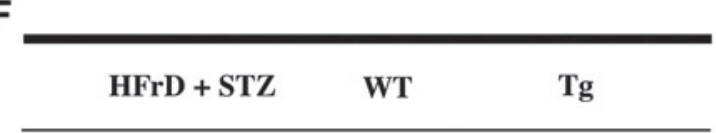

\begin{tabular}{ccc}
\hline TG in Liver $(\mathrm{mg} / \mathrm{g})$ & $18.7 \pm 2.4$ & $11.5 \pm 1.6^{*}$ \\
& & \\
FFA in Liver $(\mathrm{mEg} / \mathrm{g})$ & $13.5 \pm 3.0$ & $7.0 \pm 1.1^{*}$
\end{tabular}

D
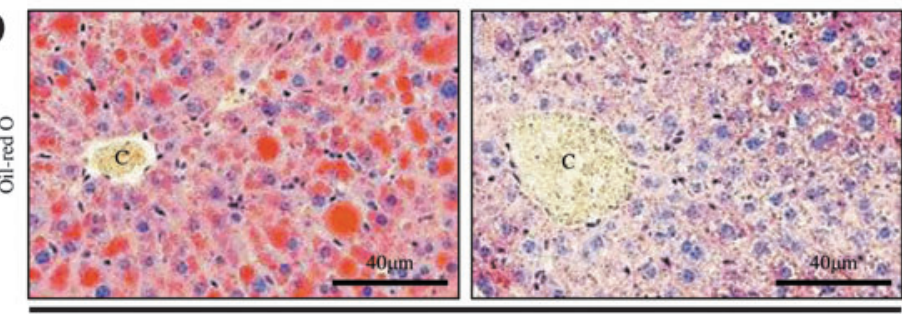

FFA in Liver $(\mathrm{mEq} / \mathrm{g}) \quad 13.5 \pm 3.0 \quad 7.0 \pm 1.1^{*}$

HFrD + STZ

FIG. 5. Histological and morphometric analyses of the liver of nongenetic mouse model. (A) Gross appearance of the NAFLD livers from mice fed the HFrD for 8 weeks and injected with STZ (HFrD +STZ, $n=10$ mice per group). The livers from WT mice were pale and yellow in color, whereas those from Tg mice showed no remarkable gross features. Scale bar $=5 \mathrm{~mm}$. (B) Representative photomicrographs of H\&E stained sections revealed lipid accumulation and inflammation particularly in WT mice after HFrD +STZ ( $n=10$ mice per group). The livers from Tg mice contained no or very few lipid droplets in hepatocytes, and no inflammatory foci. By contrast, the livers from WT mice contained significantly more lipid droplets compared with those in Tg mice. $C$, central vein; $P$, portal vein. Original magnification: $\times 40$. Scale bar $=400 \mu \mathrm{m}$. (C) High-power views of H\&E stained sections revealed macrovesicular and microvesicular steatosis throughout the entire lobules in NAFLD livers from WT mice (HFrD+STZ, $n=10$ mice per group), as well as scattered lobular and perivenular inflammation (arrowhead). Ballooning hepatocytes were occasionally seen in the WT livers (inset). C, central vein. Original magnification: $\times 200$. Scale bar $=80 \mu \mathrm{m}$. (D) Oil red-O staining confirmed increased lipid accumulation in the NAFLD livers from WT mice compared with Tg mice $(\mathrm{HFrD}+\mathrm{STZ}, n=10 \mathrm{mice}$ per group). C, central vein. Original magnification: $\times 200$, Scale bar $=40 \mu \mathrm{m}$. (E) Quantitative scoring of fat accumulation and inflammation in the NAFLD livers of WT and Tg mice (HFrD+STZ, $n=10$ mice per group). Steatosis and inflammation scores were significantly higher in the WT livers than in the Tg livers. The NASH score was also higher in the WT livers than in the Tg livers. (F) Hepatic TG and FFA contents were significantly higher in WT mice than in Tg mice (HFrD + STZ, $n=10$ mice per group). Values are means \pm SE. ${ }^{*} p<0.05,{ }^{* * *} p<0.0001$. H\&E, hematoxylin and eosin; NAFLD, nonalcoholic fatty liver disease; NASH, nonalcoholic steatohepatitis. To see this illustration in color, the reader is referred to the web version of this article at www.liebertpub.com/ars

cells positive for 8-OHdG (Fig. 8A) or 4-HNE (Fig. 8B) in Tg mice than in WT mice at 4 weeks after STZ injection (8-OHdG: WT $3.7 \pm 1.8$ vs. Tg $0.1 \pm 0.05 ; p<0.05)(4-\mathrm{HNE}$ : WT $11.1 \pm 3.8$ vs. $\operatorname{Tg} 0.4 \pm 0.2 ; p<0.05)$.

\section{Levels of serum thiobarbituric acid reactive substances in WT and Tg mice after inducing the nongenetic mouse model}

We next measured the serum levels of another oxidative stress marker, thiobarbituric acid reactive substances (TBARS).
Serum TBARS levels were significantly lower in $\mathrm{Tg}$ mice than in WT mice at 4 weeks after STZ injection (WT 41.5 $\pm 12.4 \mathrm{nmol}$ malondialdehyde ([MDA])/mg LDL protein vs. Tg $6.0 \pm$ $1.4 \mathrm{nmol} \mathrm{MDA} / \mathrm{mg}$ LDL protein, $p<0.05$ ) (Fig. 8C).

\section{Analysis of apoptotic activities after inducing the nongenetic mouse model}

Although a small number of apoptotic cells was observed in the NAFLD lesions in both WT and Tg mice, the number of terminal deoxynucleotidyl transferase end-labeling 
A
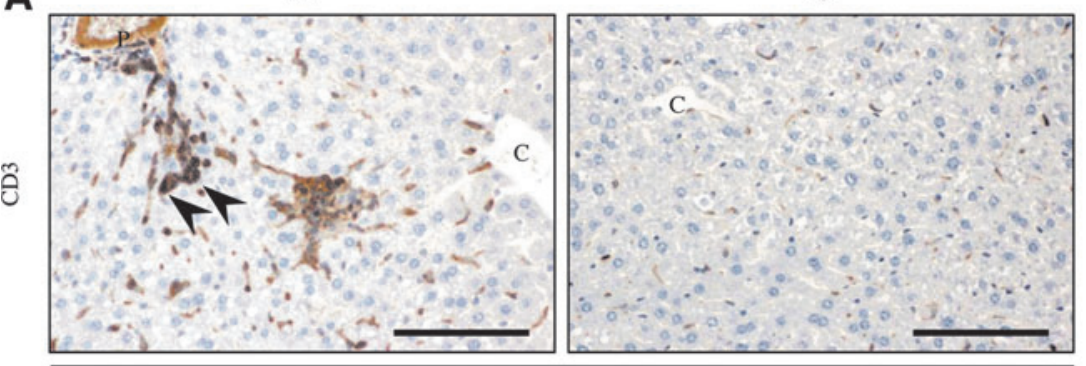

HFrD + STZ

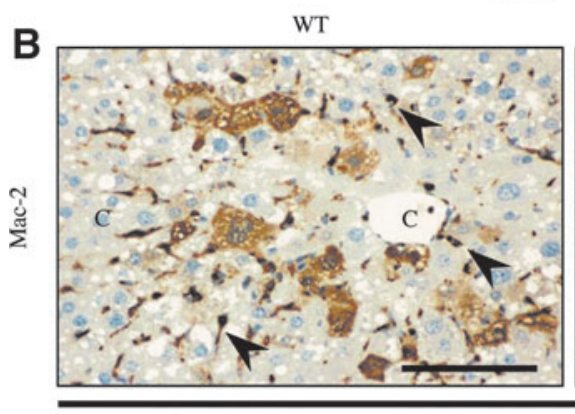

HFrD + STZ
C

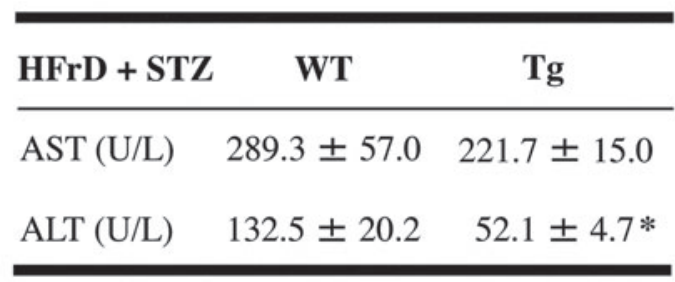

D

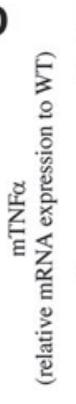

$\mathrm{Tg}$

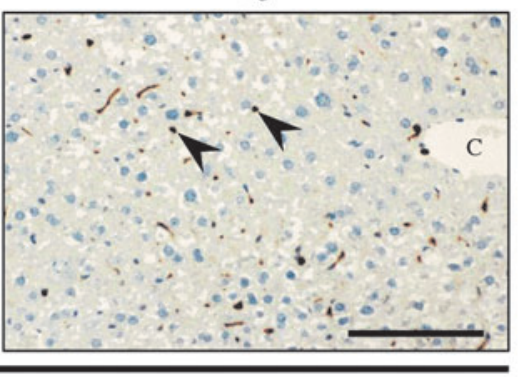

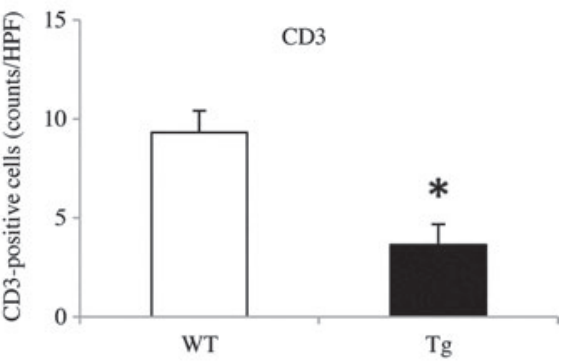

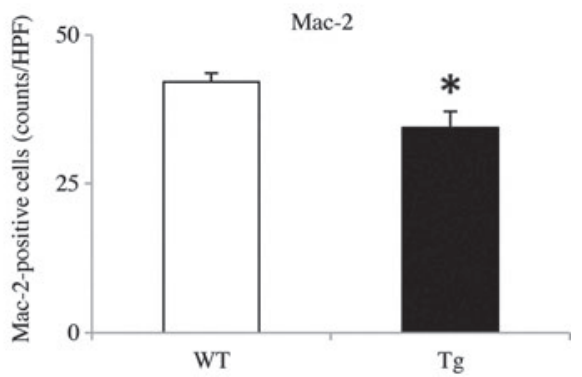

AU)

$\mathrm{mTNF} \alpha$

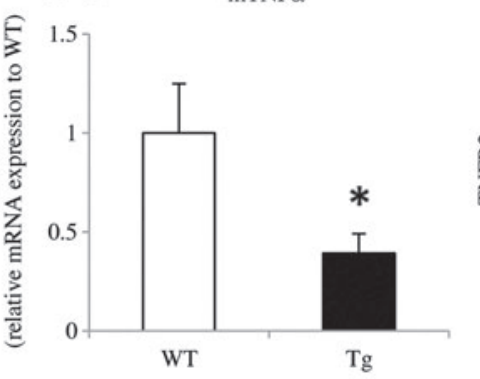

$(\mathrm{AU})$

MTNFR2

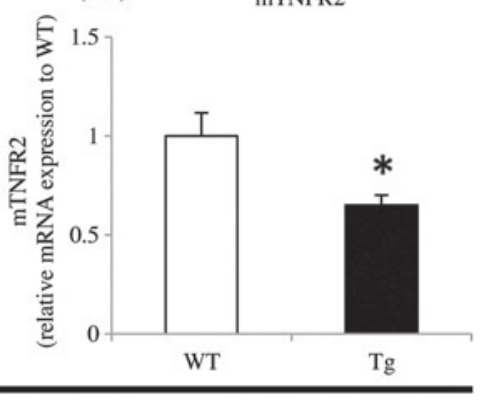

$\mathrm{HFrD}+\mathrm{STZ}$

FIG. 6. Analysis of inflammatory parameters in the liver of nongenetic mouse model. (A) IHC showed that the number of CD3-positive infiltrating $\mathrm{T}$ lymphocytes (arrowheads) in the liver was significantly smaller in Tg mice than in WT mice $(\mathrm{HFrD}+\mathrm{STZ}, n=10$ mice per group). (B) Similarly, the number of Mac-2-positive macrophages (Kupffer cells) in the liver was significantly different between WT and Tg mice (HFrD $+\mathrm{STZ}, n=10$ mice per group). (C) Plasma ALT levels, but not AST levels, were significantly lower in Tg mice than in WT mice (HFrD $+\mathrm{STZ}, n=10$ mice per group). (D) Real-time RT-PCR showed that the hepatic gene expression levels of TNF- $\alpha$ and TNFR2 were significantly downregulated in Tg mice compared with WT mice (HFrD + STZ, $n=10$ mice per group). Values are means $\pm S E$ and were normalized for $18 S$ rRNA expression (real-time RT-PCR). ${ }^{*} p<0.05$. Original magnification: $\times 200$. Scale bar $=80 \mu \mathrm{m}$. ALT, alanine aminotransferase; AST, aspartate aminotransferase; TNF, tumor necrosis factor; TNFR, tumor necrosis factor receptor; IHC, immunohistochemistry; C, central vein. To see this illustration in color, the reader is referred to the web version of this article at www.liebertpub.com/ars

(TUNEL)-positive hepatocytes was significantly lower in $\mathrm{Tg}$ mice than in WT mice (WT $0.67 \pm 0.36$ vs. Tg $0.03 \pm 0.03 ; p<0.05$ ) (Fig. 9A). Real-time RT-PCR showed that hepatic Bax and Caspase-3 expression levels were significantly lower in $\mathrm{Tg}$ mice than in WT mice (Fig. 9B, C). Furthermore, IHC analysis revealed significantly lower expression of the proapoptotic protein Bax in $\mathrm{Tg}$ mice than in WT mice (WT $1.67 \pm 0.53$ vs. Tg $0.08 \pm 0.03$, $p<0.05$; Fig. 9B); most of the Bax-positive cells could be identified as hepatocytes. IHC also revealed that the number of cleaved Caspase-3-positive hepatocytes was significantly lower in the livers of $\mathrm{Tg}$ mice than in the NAFLD livers of WT mice (WT $4.70 \pm 1.67$ vs. Tg $0.05 \pm 0.03, p<0.05$; Fig. 9C). These findings were confirmed by Western blotting analysis of Bax and activated Caspase- 3 , and the latter revealed the expression of a large 17-kDa fragment of Caspase-3 (Fig. 9B, C).

\section{Oxidative stress-induced apoptosis in cultured hepatocytes}

The nuclear morphology of cultured hepatocytes was observed after double staining with propidium iodide (red) and Hoechst 33258 (blue); TUNEL-positive apoptotic cells (green) were also analyzed in the same fields (Supplementary Fig. S4). Only a few apoptotic cells were detected in hepatocytes obtained from untreated WT and Tg mice (WT $0.36 \% \pm 0.07 \%$ vs. $\operatorname{Tg} 0.31 \% \pm 0.15 \%)$. Exposure to hydrogen peroxide $\left(\mathrm{H}_{2} \mathrm{O}_{2}\right)$ 
A
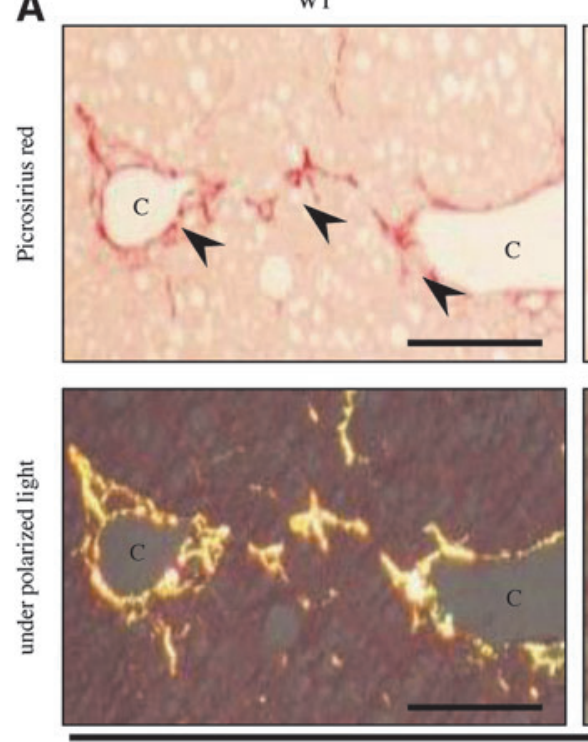

$\mathrm{HFrD}+\mathrm{STZ}$

B

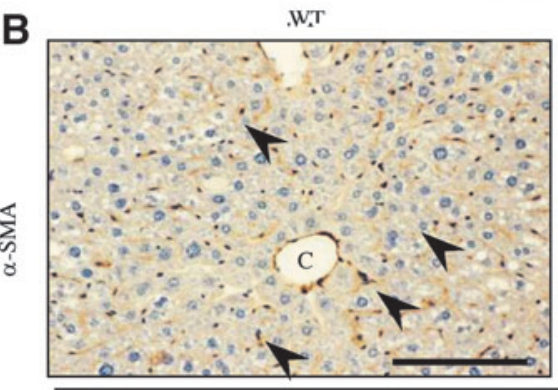

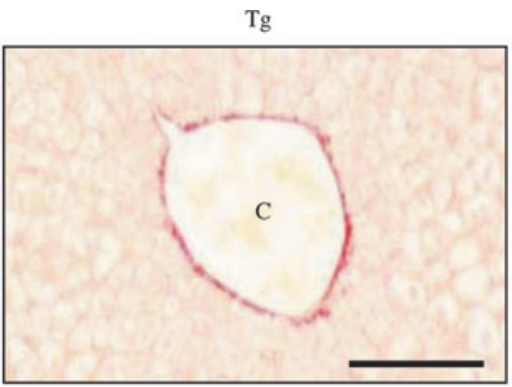

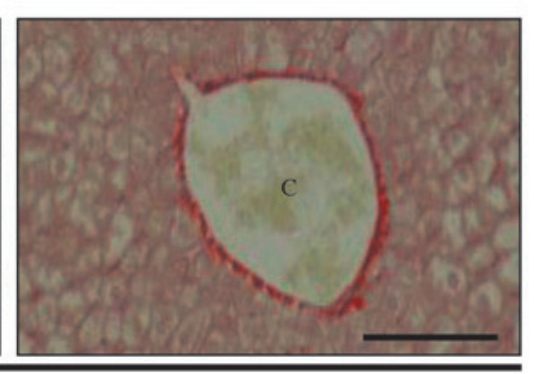

$\mathrm{Tg}$

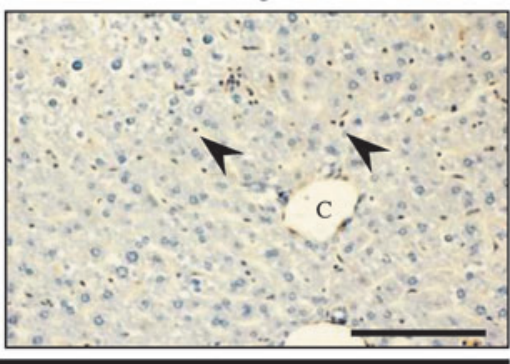

$\mathrm{HFrD}+\mathrm{STZ}$
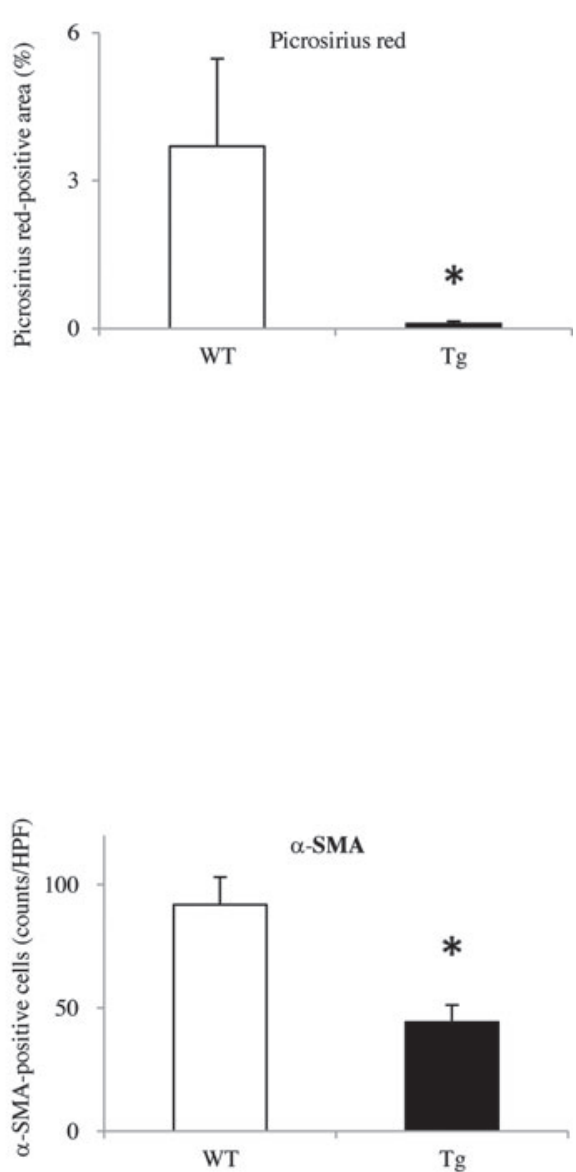

FIG. 7. Analysis of fibrogenesis in the liver of nongenetic mouse model. (A) Picrosirius red staining showed significantly larger areas of perivenular and pericellular fibrosis or fibrogenesis in the NAFLD livers of WT mice (arrowheads), compared with the Tg livers (HFrD $+\mathrm{STZ}, n=10$ mice per group). Under polarized light, the red-stained fibers were as expressing collagen type I because of yellow birefringence. (B) IHC demonstrated that there were significantly more $\alpha$-SMA-positive activated hepatic stellate cells (arrow heads) in the NAFLD livers from WT mice, mostly in picrosirius red-stained areas, than in the livers from $\operatorname{Tg}$ mice $(\mathrm{HFrD}+\mathrm{STZ}, n=10$ mice per group). Values are means \pm SE. ${ }^{*} p<0.05$. Original magnification: $\times 200$. Scale bar $=80 \mu \mathrm{m}$. C, central vein; $\alpha$-SMA, $\alpha$-smooth muscle actin. To see this illustration in color, the reader is referred to the web version of this article at www.liebertpub.com/ars

(100 $\mu$ M) significantly increased the number of apoptotic cells, although it was significantly smaller in hepatocytes from $\mathrm{Tg}$ mice than in those from WT mice $\left(\mathrm{H}_{2} \mathrm{O}_{2}\right.$ : WT $4.00 \% \pm 0.06 \%$ vs. $\operatorname{Tg} 0.74 \% \pm 0.28 \% ; p<0.0001$ ) (Supplementary Fig. S4). Some apoptotic hepatocytes showed condensed chromatin and fragmented nuclei.

\section{Expression levels of adiponectin and adiponectin receptors in WT and Tg mice in the nongenetic mouse model}

The blood adiponectin levels were significantly higher in Tg mice than in WT mice after the HFrD feeding and STZ injection for 4 weeks (WT 16.0 $\pm 0.7 \mu \mathrm{g} / \mathrm{ml} v s$. Tg 39.8 $\pm 6.6 \mu \mathrm{g} /$ $\mathrm{ml}, p<0.05$ ) (Fig. 10A). Moreover, hepatic adiponectin receptor 1 (AdipoR1) expression of mRNA levels was significantly increased in Tg mice compared with that in WT mice $(p<0.05)$, whereas that of AdipoR2 in both groups of mice showed no difference (Fig. 10B). By contrast, in the skeletal muscle, the expression levels of AdipoRs in Tg mice were also significantly upregulated $(p<0.05)$ (data not shown).

\section{Expression of PRDX4 in human between patients with T2DM and healthy adults}

ELISAs revealed that serum hPRDX4 levels were significantly higher in patients with T2DM than in a control group consisting of healthy adults (control $58.9 \pm 8.0 \mathrm{pg} / \mathrm{ml}$ vs. T2DM $131.0 \pm 21.8 \mathrm{pg} / \mathrm{ml} ; p<0.05)$ (Supplementary Fig. S5). Notably, serum hPRDX4 was significantly higher in patients with T2DM than in Tg mice following induction of the nongenetic mouse model, as described above $(\operatorname{Tg} 22.8 \pm 4.0 \mathrm{pg} / \mathrm{ml}$ vs. T2DM $131.0 \pm 21.8 \mathrm{pg} / \mathrm{ml} ; p<0.05$ ) (Fig. 2D).

\section{Discussion}

This study for the first time revealed the possible mechanisms by which PRDX4 may protect against NAFLD and T2DM. Overexpression of intracellular and secreted PRDX4 (i) suppressed local (intrahepatic) and systemic (circulating) expression of oxidative stressors; (ii) reduced steatosis (and possibly obesity), inflammation, and apoptosis; and (iii) ultimately improved insulin resistance and NASH through these 
A
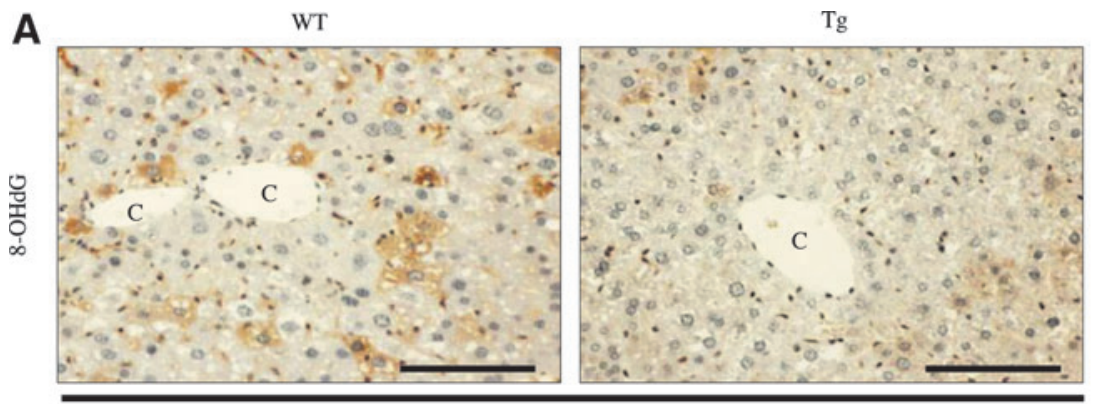

$\mathrm{HFrD}+\mathrm{STZ}$
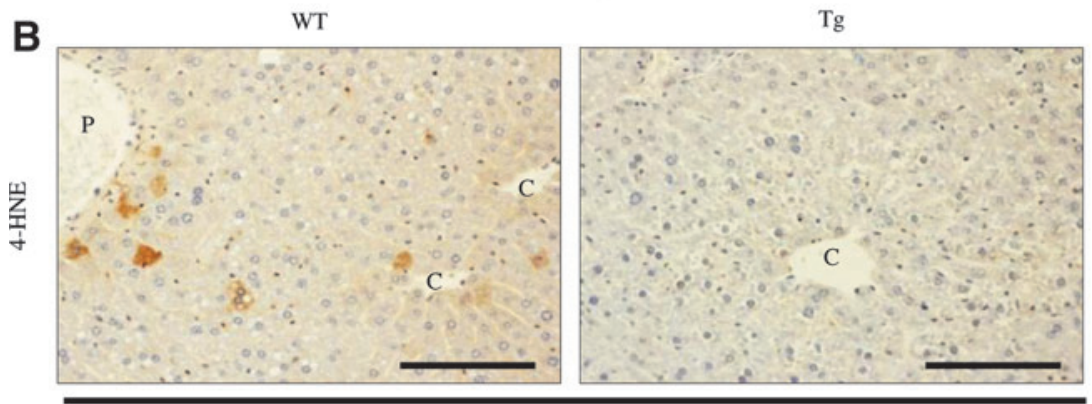

$\mathrm{HFrD}+\mathrm{STZ}$
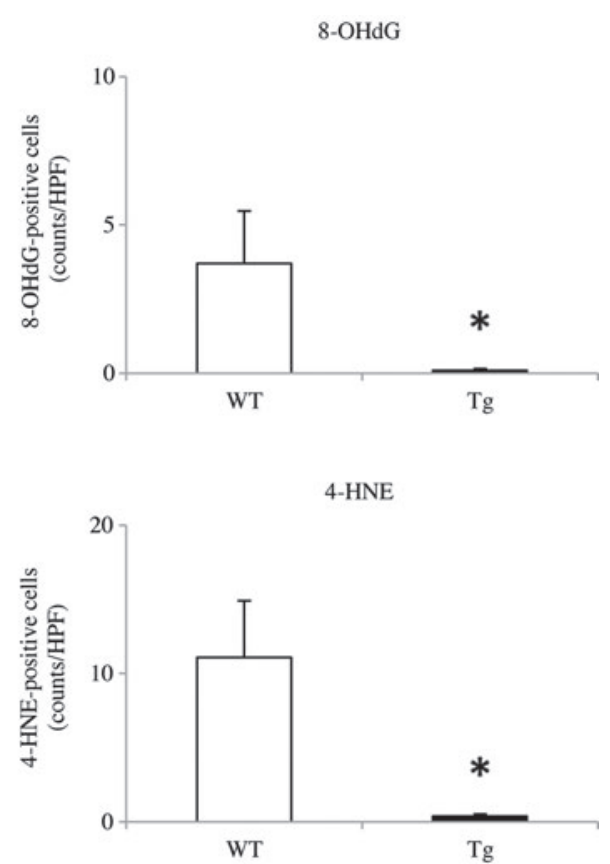

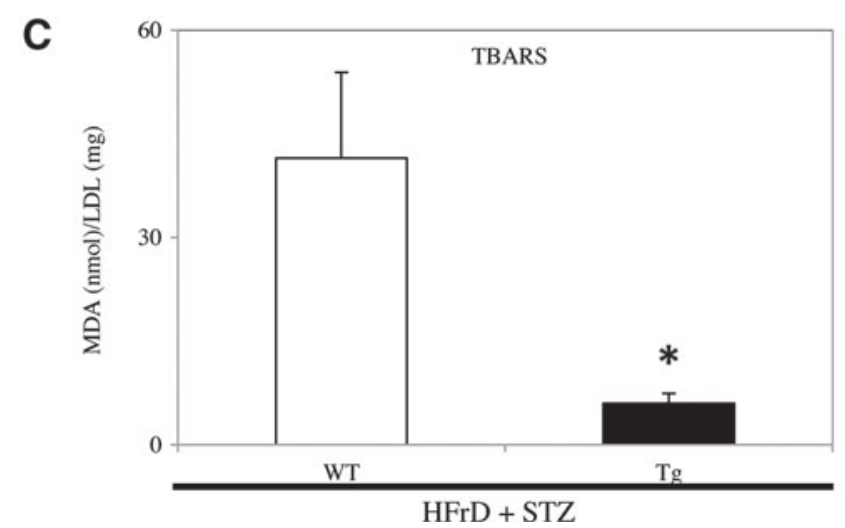

FIG. 8. Analysis of oxidative stress markers in the nongenetic mouse model. (A, B) IHC showed increased expressions of 8-OHdG (A) and 4-HNE (B) in mice fed the HFrD and injected with STZ (HFrD + STZ, $n=10$ mice per group). The numbers of 8-OHdG- and 4-HNE-positive hepatocytes (arrowheads) were significantly greater in the NAFLD livers from WT mice than in the livers from Tg mice. ${ }^{*} p<0.05$. C, central vein. Original magnification: $\times 200$. Scale bar $=80 \mu$ m. (C) Serum TBARS levels expressed as nmol MDA/mg LDL protein were significantly lower in Tg mice than in WT mice after 8 weeks of HFrD feeding and STZ injection (HFrD+STZ, $n=10$ mice per group). ${ }^{*} p<0.05$. Values are means \pm SE. 4-HNE, 4-hydroxy-2-nonenal; 8OHdG, 8-hydroxy-2'-deoxyguanosine; MDA, malondialdehyde; TBARS, thiobarbituric acid reactive substances. To see this illustration in color, the reader is referred to the web version of this article at www.liebertpub.com/ars

effects. Supplementary Figure S6 summarizes the roles of PRDX4 in the current nongenetic mouse model of NAFLD and T2DM.

Interestingly, after establishing the current nongenetic mouse model, we detected specific expression of hPRDX4 in the liver of Tg mice by PCR, Western blotting, and IHC (Figs. $1 \mathrm{~A}, \mathrm{~B}$ and $2 \mathrm{~B}, \mathrm{C}$ ), but not or faint (indicating cross-reactivity of the antibody against hPRDX4 to endogenous mPRDX4) in the NASH-like livers of WT mice (Fig. 2B, C). It seems feasible that the cytomegalovirus enhancer/promoter is stimulated by ROS and inflammation during the progression of NAFLD in this model via cross talk with other promoters for other transcription factor-binding sites such as nuclear factor-kappa $B(7,20)$. This should increase the expression of activated antioxidant proteins, including transgenic hPRDX4 and endogenous mPRDX4 (Fig. 1). We also observed that the expression of this transgene overcame that of the endogenous gene in the modest NAFLD livers of Tg mice (Fig. 1A). The circulating serum levels of the soluble hPRDX4 protein were also significantly elevated in Tg mice (Fig. 2D), presumably in addition to the changes in mPRDX4. These results suggest that the establishment of insulin resistance and NAFLD in the present model increases oxidation by increasing hepatic and blood ROS levels, as evidence by IHC of 8-OHdG or 4-HNE in hepatocytes and measurement of circulating TBARS, respectively (Fig. 8). These findings indicate that Tg mice provide a good model for determining the local (i.e., intracellular) and systemic (i.e., circulating) effects of increased hPRDX4 and endogenous mPRDX4 expression on HFrD plus STZ-induced T2DM, NASH, and possibly the metabolic syndrome. In fact, 
A
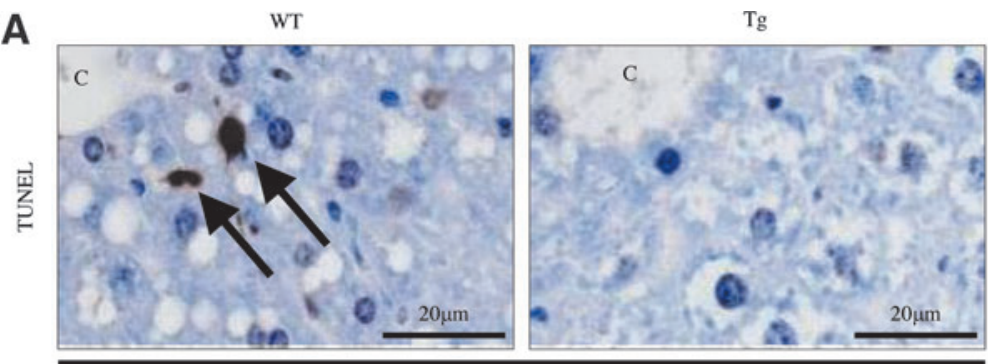

HFrD + STZ

B
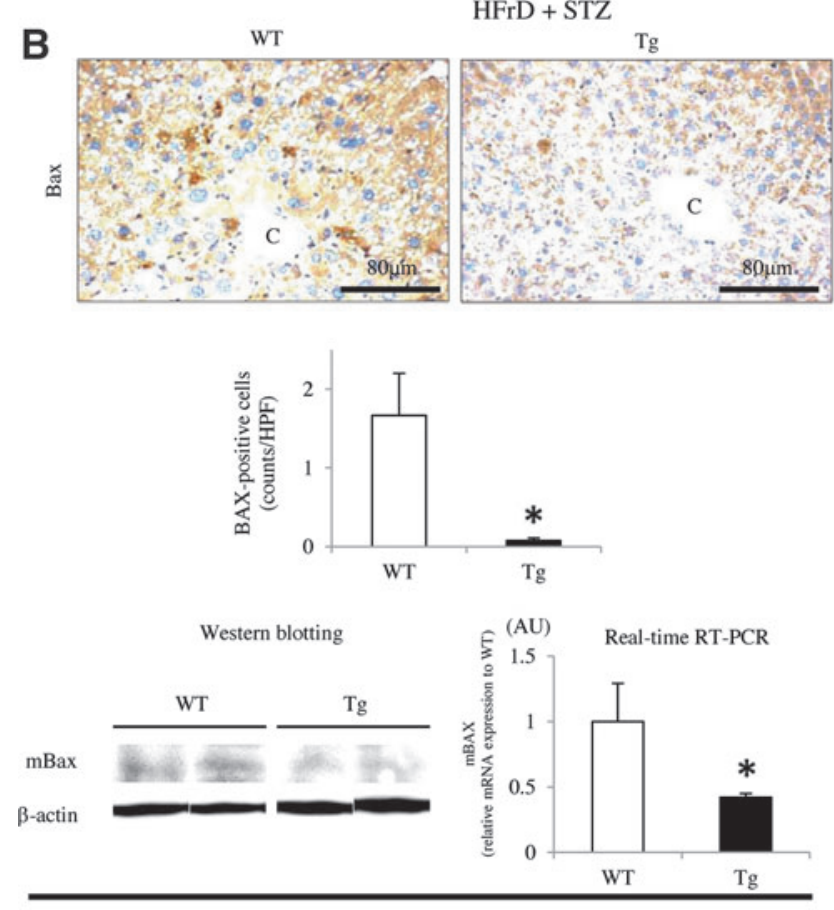

$\mathrm{HFrD}+\mathrm{STZ}$

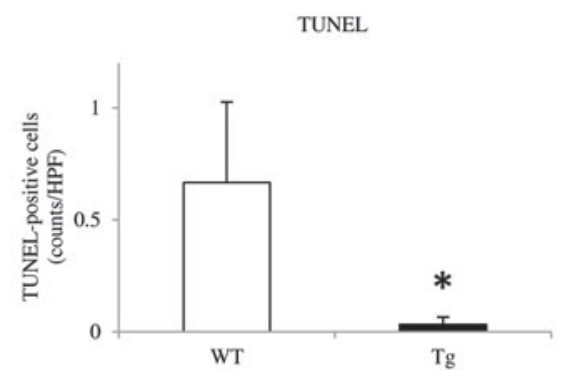

C
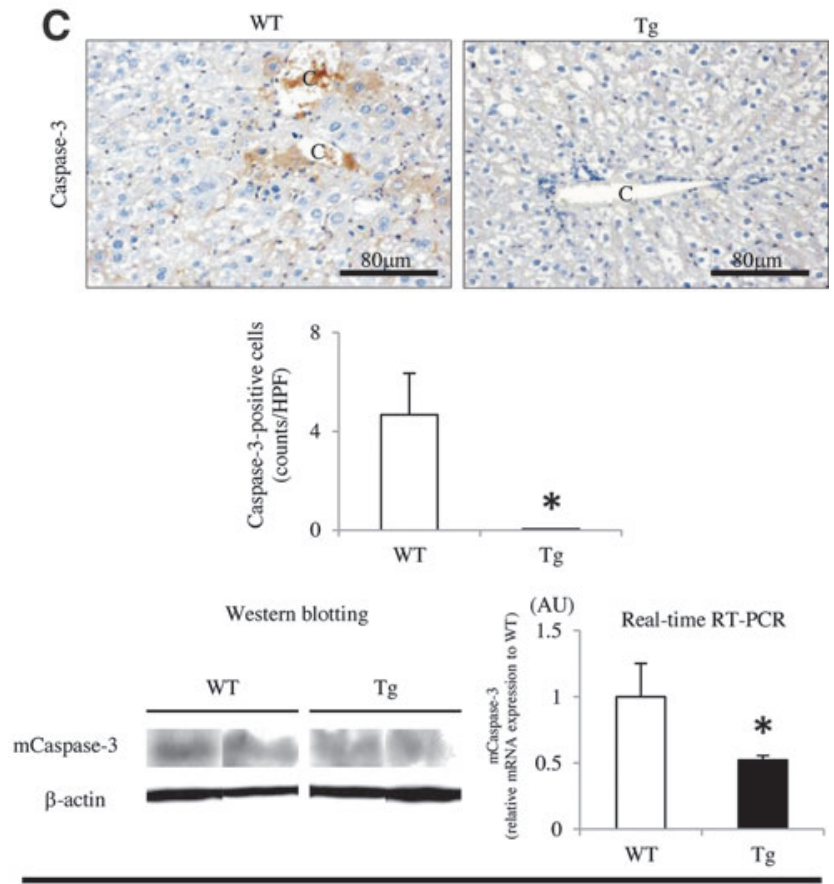

$\mathrm{HFrD}+\mathrm{STZ}$

FIG. 9. Hepatic apoptotic activity in the nongenetic mouse model. (A) The number of TUNEL-positive hepatocytes in NAFLD lesions was significantly lower in Tg mice than in WT mice (arrows) $(\mathrm{HFrD}+\mathrm{STZ}, n=10$ mice per group). $\mathrm{C}=$ central vein. Original magnification: $\times 400$. Scale bar $=20 \mu \mathrm{m}$. (B) IHC and Western blotting showed that hepatic Bax protein expression was significantly decreased in Tg mice than in WT mice (HFrD $+\mathrm{STZ}, n=10$ mice per group). Real-time RT-PCR revealed that hepatic Bax mRNA expression was significantly lower in Tg mice than in WT mice $(\mathrm{HFrD}+\mathrm{STZ}, n=10 \mathrm{mice}$ per group). C, central vein. Original magnification: $\times 200$. Scale bar $=80 \mu \mathrm{m}$. (C) Similarly, IHC (original magnification: $\times 200$. Scale bar $=80 \mu \mathrm{m}$.), Western blotting, and real-time RT-PCR showed that the hepatic expression of the large fragment of activated caspase-3 $(17 \mathrm{kDa})$ was significantly lower in $\mathrm{Tg}$ mice than in WT mice (HFrD+STZ, $n=10$ mice per group). Values are means \pm SE and were normalized for $18 S$ rRNA expression (real-time RT-PCR) or $\beta$-actin expression (Western blotting). ${ }^{*} p<0.05$. TUNEL, terminal deoxynucleotidyl transferase end-labeling; $\mathrm{C}$, central vein. To see this illustration in color, the reader is referred to the web version of this article at www.liebertpub.com/ars

the amino acid sequence of mPRDX4 shows very high homology to that of hPRDX4 (>89\%), as determined by the Basic Local Alignment Search Tool (BLAST; National Center for Biotechnology Information, U.S. National Library of Medicine, Bethesda, MD).

After STZ injection, WT mice showed gradual, but dramatic increases in blood glucose and insulin levels, as well as delayed glucose clearance (Fig. 3A-C). This apparent dysfunction of glucose/insulin metabolism may be attributed to the significant reduction in insulin sensitivity, especially in the liver, because of increased TG and FFA accumulation, manifesting as NAFLD, rather than disruption of pancreatic islets by STZ-induced insulitis. This is because the present nongenetic mouse model was established using a relatively low dose of STZ, unlike T1DM models in which a single high dose of STZ is used (5). Indeed, there were no morphological differences between the islets of mice treated with or without STZ (Supplementary Fig. S1B). Notably, Tg mice had significantly lower glucose and insulin levels, together with faster glucose clearance during the glucose tolerance test (Fig. 3AC). Furthermore, Tg mice had higher adiponectin levels, together with higher expression levels of hepatic and muscular AdipoRs (Fig. 10). As adiponectin is an anti-steatogenic adipocytokine that can decrease the hepatic lipid content and protect against liver fibrosis $(27,31)$, these findings indicate that insulin sensitivity is enhanced in the peripheral tissues of $\mathrm{Tg}$ mice, particularly in the liver and skeletal muscle, compared with WT mice. We also fed Tg and WT mice with the HFrD without STZ injection (Supplementary Fig. S2). However, after 20 weeks of feeding, we did not detect any 


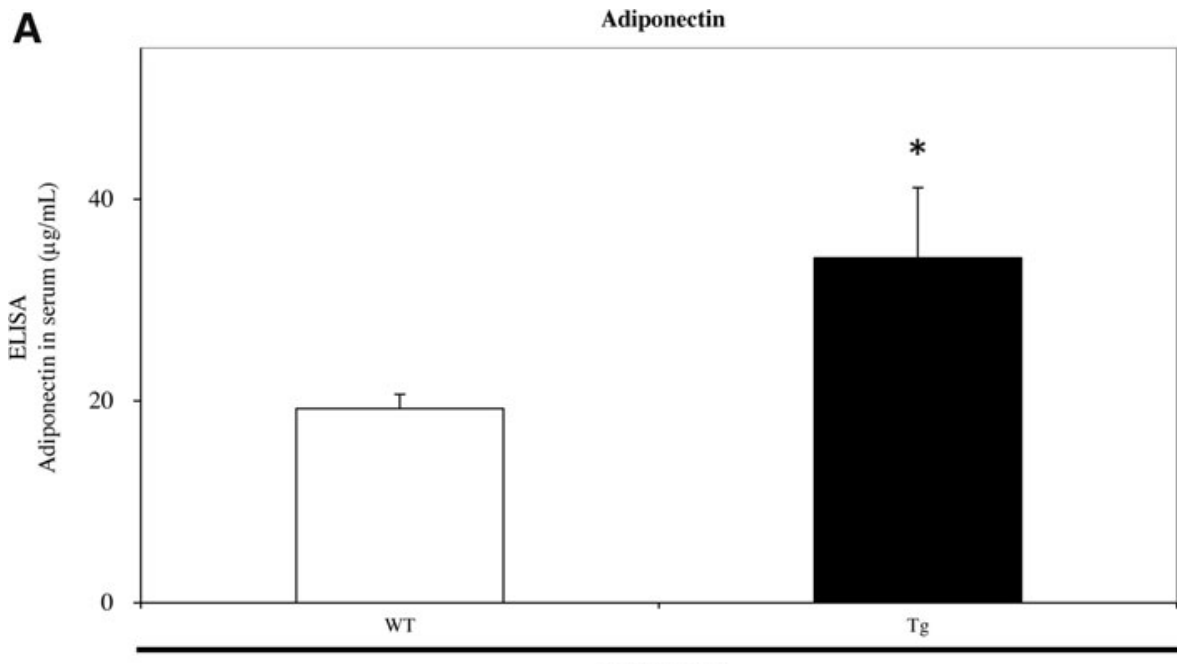

$\mathrm{HFrD}+\mathrm{STZ}$

B

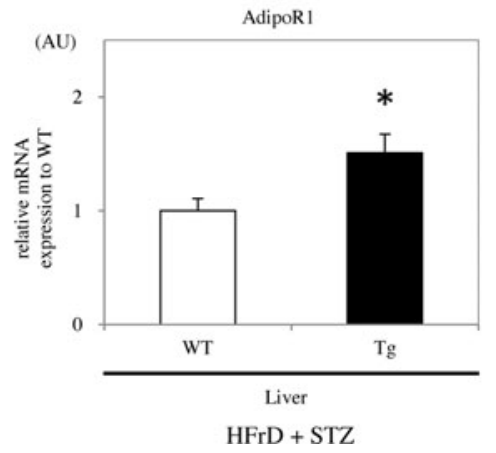

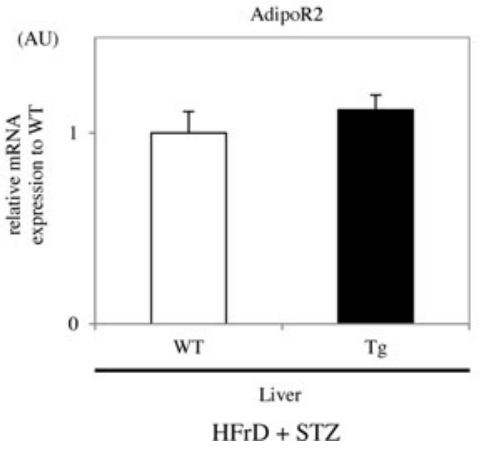

FIG. 10. Adiponectin and AdipoRs expression in the nongenetic mouse model. (A) Blood adiponectin levels were significantly higher in $\mathrm{Tg}$ mice than in WT mice after being fed the HFrD for 8 weeks and injected with STZ (HFrD+STZ, $n=10$ mice per group). (B) Real-time RT-PCR showed that hepatic AdipoR1 expression was significantly greater in $\mathrm{Tg}$ mice than in WT mice, whereas AdipoR2 was not significantly different (HFrD+STZ, $n=10$ mice per group). Values are means \pm SE and were normalized for $18 S$ rRNA expression (real-time RTPCR). ${ }^{*} p<0.05$. AdipoR, adiponectin receptor. significant differences in terms of glucose levels or liver morphology, except for visceral obesity, between WT and Tg mice, unlike in mice given the $\mathrm{HFrD}$ plus STZ injection. Although this discrepancy between these two models cannot be explained, the nongenetic mouse model induced by HFrD plus STZ injection seems to be very appropriate to study the protective effects of PRDX4 against NASH-associated insulin resistance in vivo. Nevertheless, it is possible that PRDX4 also protects against obesity. As the second NASH model induced by a MCD plus HF diet (15) confirmed the protective effects of PRDX4 overexpression against NAFLD progression, these effects of PRDX4 might not be specific to the animal models used here. The features of liver from $m P R D X 4^{-/ y}$ mice (9) provide further support for the protective effects of PRDX4 against NASH (Supplementary Fig. S3). Therefore, we would like to study the relationship between PRDX4 knockdown and metabolic syndrome in this animal model in the near future.

The serum levels of lipoproteins were surprisingly lower in $\mathrm{Tg}$ and WT mice of the nongenetic mouse model, while T-cho, LDL, and HDL levels were significantly higher and TG levels were significantly lower in Tg mice than in WT mice (Fig. 4D). In these HFrD plus STZ-induced model mice, serum lipoprotein levels, including TG might not significantly affect the progression of NAFLD, unlike hepatic TG accumulation. To the best of our knowledge, no reports have examined the histopathological features of the liver or of serum lipoprotein levels in this animal model. Therefore, the correlation between serum lipoprotein profiles and PRDX4 overexpression remains unknown, and this issue should be examined in more detail after accumulating further experimental data.

NASH differs from simple steatosis in terms of the degree of hepatic injury, inflammation, and apoptosis, and its pathogenesis involves the widely accepted "two-hit" hypothesis $(4,25)$. In the current study, morphologic, biochemical, and molecular analyses showed that macrovesicular steatosis, as well as TG and FFA accumulation, were significantly suppressed in the liver of Tg mice (Fig. 5). These findings were accompanied by fewer $\mathrm{T}$ lymphocytes and Kupffer cells, a lower hepatic aminotransferase level (Fig. 6A-C), and reduced expression of TNF- $\alpha$ and its receptor (Fig. 6D), compared with those in the NASH-like liver of WT mice. Furthermore, local 4-HNE and 8-OHdG expression, and circulating TBARS levels, as markers of oxidative stress $(5,7)$, were reduced in Tg mice (Fig. 8). Thus, the present findings imply that overexpression of hPRDX4 in the modest NAFLD liver of $\mathrm{Tg}$ mice protects against chronic inflammation and subsequent disease progression, at least partly by scavenging extracellular ROS and preventing inflammatory cell-derived ROS generation, consistent with our previous reports $(5,7)$. The present results also indicate that PRDX4 suppresses the progression of simple steatosis to NASH in C57BL/ 6 mice fed a HFrD and injection with low-dose STZ by blocking the first hit (disordered hepatic lipid accumulation) and the second hits (metabolic, cytokine, and oxidative stressors), and should protect against the initiation and/or progression of T2DM.

As apoptosis sometimes triggers aberrant regenerative responses, resulting in uncontrolled activation and differentiation 
of stellate cells into myofibroblasts and subsequently liver fibrogenesis, it is possible that apoptotic hepatocytes provide a critical "third hit" that may drive the progression from NASH to cirrhosis (25). In fact, in vivo, there were significantly fewer apoptotic hepatocytes in the Tg livers, which was accompanied by lower expression levels of the proapoptotic markers, Bax and cleaved Caspase-3 (Fig. 9), compared to those in the NASH-like livers of WT mice. Our subsequent in vitro studies strongly supported these results, as oxidative stress $\left(\mathrm{H}_{2} \mathrm{O}_{2}\right)$-induced apoptosis was markedly attenuated in cultured hepatocytes obtained from Tg mice (Supplementary Fig. S4). Thus, we provide the first evidence demonstrating that overexpression of hPRDX4 suppressed hepatocyte apoptosis and that oxidative stress is closely associated with apoptosis (7). In addition to the antiapoptotic effects, Tg liver showed significantly reduced expression of $\alpha$-SMA, a marker of stellate cell activation, and of collagen type I, a marker of hepatic fibrosis/fibrogenesis (Fig. 7). Therefore, it seems that PRDX4 also attenuates the progression of NASH to cirrhosis by suppressing hepatocyte apoptosis (i.e., the "third hit") and liver fibrosis. Therefore, PRDX4 might protect against carcinogenesis as well as metabolic syndrome, including T2DM and/or NASH.

In conclusion, $\mathrm{Tg}$ mice were protected from the HFrD and STZ-induced NAFLD and insulin resistance through the overexpression of hPRDX4, particularly in the liver. Our data indicate that hPRDX4 overexpression plays important roles in (i) ameliorating the initiation of NAFLD and insulin resistance by reducing hepatic TG and FFA accumulation, and (ii) preventing the development of NASH and T2DM by suppressing inflammation, apoptosis, and fibrosis, with local (intracellular or intrahepatic) and systemic (circulating) effects, as summarized in Supplementary Figure S6. Our observations confirm that $h P R D X 4 \mathrm{Tg}$ mice could be a useful animal model to study the associations of oxidative stress and with inflammation and glucose/lipid metabolism. Our findings also suggested that activators of PRDX4 could offer a potential therapeutic agent for ameliorating NASH and/or T2DM by suppressing oxidative damage and inflammatory signaling and by improving liver insulin sensitivity throughout the course of these diseases. Indeed, we found that patients with T2DM have significantly higher serum hPRDX4 levels than healthy adult volunteers (Supplementary Fig. S5), possibly because of the greater demand for antioxidant defense in T2DM. Therefore, PRDX4 might have pathophysiological relevance for protecting against metabolic syndrome in humans.

\section{Materials and Methods}

Detailed Materials and Methods are given in the Supplementary Data.

\section{Construction of the $\mathrm{Tg}$ vector}

hPRDX4 cDNA was amplified by RT-PCR and cloned into the pGEM-T easy vector system (Invitrogen, Life Technologies Japan Ltd., Tokyo, Japan), as previously described (Supplementary Fig. S1A) $(5,7,29)$.

\section{Animals and establishment of NAFLD and T2DM}

Experiments were performed using 4-6-week-old male WT and $\mathrm{Tg}$ mice weighing $\sim 18 \mathrm{~g}$, maintained in a temperature- and light-controlled facility with free access to water. Mice were fed a HFrD (67\% carbohydrates-98\% of which is fructose-, $13 \%$ fat, and 20\% protein; KBT Oriental Corporation, Saga, Japan) for 4 weeks to generate peripheral insulin resistance and NAFLD followed by a single intraperitoneal injection of $100 \mathrm{mg} / \mathrm{kg}$ BW of freshly prepared STZ (SigmaAldrich, Co., St Louis, MO) dissolved in a sodium citrate buffer ( $\mathrm{pH} 4.5$ ) after fasting for $18 \mathrm{~h}$. The mice were then fed the same HFrD for a further 4 weeks in a nongenetic mouse model, as previously described (17). At 1, 2, 3, and 4 weeks after STZ injection, tail vein blood glucose levels were measured using a blood glucose monitor (Sanwa Kagaku Kenkyusho Co., Ltd., Nagoya, Japan), and mice with blood glucose levels $>250 \mathrm{mg} / \mathrm{dl}$ were considered to be diabetic. Animals were killed 4 weeks after STZ injection by intraperitoneal anesthetization with an overdose of ketamine (100 mg/kg) (Daiichi Sankyo Co., Tokyo, Japan) and medetomidine (2 mg/kg) (Meiji Yakuhin Co., Tokyo, Japan), and tissues, including the liver, pancreas, and WAT were excised. As additional animal models, 8-week-old male WT and Tg mice were fed the HFrD for 20 weeks or a diet (MCD + HF) for 4 weeks, as previously described (15); STZ was not injected in these two models. Blood glucose concentrations, BW, organ weights, and food intakes were measured every week, or when killed. Food consumption was determined in metabolic cages obtained from Sugiyama-Gen Co., Ltd. (Tokyo, Japan).

All protocols were approved by the Ethics Committee of Animal Care and Experimentation, University of Occupational and Environmental Health, Japan, and were performed according to the Institutional Guidelines for Animal Experiments and the Law (no. 105) and Notification (no. 6) of the Japanese Government. The study also conforms to the Guide for the Care and Use of Laboratory Animals published by the US National Institutes of Health (NIH Publication No. 85-23, revised 1996).

\section{Histology and IHC}

A rabbit anti-human polyclonal antibody (1:500; Affinity BioReagents, Golden, CO) was used to detect hPRDX4. To our knowledge, there are no specific antibodies available for endogenous mPRDX4. For histological analyses of the liver, images of H\&E, Oil Red-O, picrosirius red, or IHC sections were captured and quantified using NanoZoomer Digital Pathology Virtual Slide Viewer software (Hamamatsu Photonics Corp., Hamamatsu, Japan) $(5,7)$. To evaluate the degree of lipid accumulation (steatosis score), we performed Oil Red-O staining using paraffin-embedded liver sections fixed in osmium tetroxide and categorized the tissue into four grades, as follows (27): no lipid droplets (score $=0$ ); lipid droplets in $<33 \%$ of hepatocytes (score $=1$ ); lipid droplets $33 \%-66 \%$ of hepatocytes (score $=2$ ); and lipid droplets in $>66 \%$ of hepatocytes (score $=3$ ).

To determine ROS/oxidative stress or expression in hepatocytes after STZ injection, we used an 8-OHdG monoclonal antibody (1:100; Japan Institute for the Control of Aging, Fukuroi, Japan) or a 4-HNE monoclonal antibody (1:100; Japan Institute for the Control of Aging) and quantified the number of hepatocytes positive for either antibodies in five randomly selected fields per section (original magnification: $\times 200)$, as previously described $(5,7,30)$. The endogenous peroxidase activity was not blocked in analysis of oxidative 
stress markers $(5,7)$. To evaluate the severity of NASH, we determined the intensity of inflammation (inflammatory score), using a polyclonal rabbit anti-human CD3 antibody (1:1; Dako Cytomation Co., Tokyo, Japan) or an anti-mouse Mac-2 monoclonal antibody (1:500; Cedarlane Laboratories Ltd., Burlington, Ontario, Canada). We counted the number of positive T lymphocytes or macrophages (Kupffer cells) in 10 randomly selected fields per section (original magnification: $\times 200)$. The tissues were then classified into four grades, as follows (27): no inflammation (score $=0)$; $<10$ inflammatory foci, each consisting of $>5$ inflammatory cells (score $=1$ ); $\geq 10$ inflammatory foci (score $=2$ ); or uncountable diffuse or fused inflammatory foci (score $=3$ ). Hepatocyte apoptosis was determined by staining tissue sections with the Bax rabbit polyclonal antibody (1:50; Santa Cruz Biotechnology, Santa Cruz, CA) and the Caspase-3 rabbit monoclonal antibody (1:25; Epitomics, Inc., Burlingame, CA). The number of apoptotic cells was counted in 10 randomly selected fields per section (original magnification: $\times 200$ ), as previously described $(5,7)$.

To assess activation of stellate cells in NAFLD lesions, we first used the HistoMouse ${ }^{\mathrm{TM}}-\mathrm{Plus}$ Kit (Invitrogen Corporation, Camarillo, CA) to block endogenous IgG, and then stained tissue with a monoclonal mouse anti-human $\alpha$-SMA antibody (1:1000; Dako Cytomation Co.). The number of activated stellate cells was then counted in 10 randomly selected fields per section (original magnification: $\times 200$ ), as previously described (7). Liver fibrosis was quantified by picrosirius red staining (Picrosirius Red Stain Kit; Polysciences, Inc., Warrington, PA) in 10 randomly selected fields per section (original magnification: $\times 200$ ). Fibers stained red with yellow birefringence under polarized light were defined as expressing collagen type I. For IHC studied, we examined one section from each of 10 mice per experimental group at least. All histological and IHC slides were evaluated by two independent observers (certified pathologists-Sohsuke Yamada and Shohei Shimajiri) who were blinded to the physical outcome or other biological and pathological data for each sample. In case of disagreement, a consensus score was determined by a third board-certified pathologist (Yasuyuki Sasaguri).

To evaluate the degree of insulitis or visceral obesity, we calculated the mean pancreatic islet area or adipocytes in five randomly selected fields per section using NanoZoomer Digital Pathology Virtual Slide Viewer software (Hamamatsu Photonics Corp.) (original magnification: $\times 200$ ), as previously described $(5,7)$.

\section{TUNEL staining}

To determine the number of apoptotic cells in NAFLD lesions, we conducted TUNEL assays using the in situ Cell Death Detection Kit, POD (Roche Applied Science, Lewes, United Kingdom) $(5,7)$.

\section{Measurement of TBARS levels}

The measurement of TBARS levels is a well-established method to evaluate lipid peroxidation (7). Therefore, as a second quantitative marker of oxidative stress that is closely associated with the development of NASH, we measured serum TBARS levels using a TBARS Assay Kit (Cayman
Chemical Company, Ann Arbor, MI). Results are expressed as $\mathrm{nmol} \mathrm{MDA} / \mathrm{mg}$ LDL protein.

\section{Analyses of hepatic injury and lipid content}

Serum levels of hepatic injury-related enzymes, including AST and ALT, were measured using commercial assay kits (Wako Pure Chemical Co., Osaka, Japan). To examine the hepatic lipid profile, the snap-frozen liver tissue $(30 \mathrm{mg})$ was homogenized and extracted with the chloroform-methanol $(2 / 1 \mathrm{v} / \mathrm{v})$ solution, as previously described (27). The organic phase was dried and resolubilized in 2-propanol. Then, the contents of FFA and TG were determined using commercial assay kits (Wako Pure Chemical Co.).

\section{HPLC analysis of lipoprotein}

After the HFrD feeding for 8 weeks with STZ injection, the mice were fasted for $7 \mathrm{~h}$ and blood was collected from the axillary artery into a microtube containing $5 \mu \mathrm{l}$ of $0.5 \mathrm{~mol} / \mathrm{L}$ EDTA. Samples were centrifuged for $12 \mathrm{~min}$ at $5500 \mathrm{~g}$ at $4^{\circ} \mathrm{C}$ and the resulting serum was stored at $-80^{\circ} \mathrm{C}$ until assayed. Lipoproteins were analyzed by HPLC using molecular sieve columns (Skylight Biotech, Inc., Akita, Japan), as previously described $(7,27)$.

\section{$R T-P C R$ and real-time $R T-P C R$}

RT-PCR and real-time RT-PCR were used to analyze gene expression in the liver and skeletal muscle, as previously described $(5,7,27)$. The primers and probes used for each gene are listed in Supplementary Table SI. The relative expression levels of each gene were normalized for those of glyceraldehydes 3-phosphate dehydrogenase (GAPDH) and 185 ribosomal RNA (rRNA) using random primers, as previously described $(5,7)$.

\section{Western blotting}

Liver protein $(40 \mu \mathrm{g})$ samples were separated by electrophoresis on $10 \%$ SDS-PAGE gels and transferred onto Immun-Blot PVDF membranes (Bio-Rad Laboratories, K.K., Tokyo, Japan). The membranes were then incubated with the rabbit anti-human polyclonal hPRDX4 antibody (1:10,000; Affinity BioReagents), rabbit anti-mouse monoclonal cleaved Caspase-3 (1:125; Epitomics, Inc.), rabbit anti-mouse polyclonal Bax (1:100; Santa Cruz Biotechnology), and the mouse anti-chicken monoclonal $\beta$-actin antibody (1:1000; Santa Cruz Biotechnology).

\section{hPRDX4, insulin, and adiponectin ELISAs}

Serum hPRDX4, insulin, and adiponectin levels at 0 and 4 weeks after STZ injection were measured using commercial ELISAs (hPRDX4: Abnova, Taipei, Taiwan; insulin: Morinaga Institute of Biological Science, Inc., Yokohama, Japan; and adiponectin: R\&D Systems, Minneapolis, MN).

\section{Glucose tolerance and glucose response test}

To determine glucose tolerance, mice were fasted for $18 \mathrm{~h}$ and given an intraperitoneal injection of glucose ( $1 \mathrm{mg} / \mathrm{g} \mathrm{BW}) 3$ days before being killed. For the glucose response to insulin, mice received an intraperitoneal injection of insulin $(1 \mathrm{U} / \mathrm{kg} \mathrm{BW})$ 
1 day before being killed. Tail vein blood samples were collected at $0,30,60$, and $120 \mathrm{~min}$ after glucose or insulin injection (5).

\section{Statistical analysis}

Results are expressed as means \pm SE. Significant differences were analyzed using the Student's $t$-test, Welch's $t$-test, or one-way analysis of variance (ANOVA) where appropriate. In all cases when ANOVA methodology was employed, the Tukey's multiple comparison post hoc test was used. Values of $p<0.05$ were considered to be statistically significant.

\section{Acknowledgments}

We would like to thank Hiroko Isagai, Hana Nishimura, and Naoko Une for their expert technical assistance; we thank Masaru Harada, Mitsuyoshi Yamamoto, and Masashi Taguchi (Third Department of Internal Medicine, University of Occupational and Environmental Health, School of Medicine), and Shinji Matsumoto, for collecting and preparing from peripheral blood cells of patients with T2DM; and we also thank Sachiko Iwai (Information, Production and Systems Research Center, Waseda University) for her expert assistance with statistical analysis. This work was supported, in part, by Grants-in-Aid for Scientific Research (24790394, 20590416, and 19590413) from the Ministry of Education, Culture, Sports, Science, and Technology, Tokyo, Japan (to S.Y., Y.S., K.K., and A.T.).

\section{Author Disclosure Statement}

No competing financial interests exist.

\section{References}

1. Agarwal $\mathrm{N}$ and Sharma BC. Insulin resistance and clinical aspects of non-alcoholic steatohepatitis (NASH). Hepatol Res 33: 92-96, 2005.

2. Angulo P. Nonalcoholic fatty liver disease. N Engl J Med 346: 1221-1231, 2002.

3. Busserolles J, Gueux E, Rock E, Demigné C, Mazur A, and Rayssiguier Y. Oligofructose protects against the hypertriglyceridemic and pro-oxidative effects of a high fructose diet in rats. J Nutr 133: 1903-1908, 2003.

4. Day CP and James OF. Steatohepatitis: a tale of two "hits"? Gastroenterology 114: 842-845, 1998.

5. Ding Y, Yamada S, Wang KY, Shimajiri S, Guo X, Tanimoto A, Murata Y, Kitajima S, Watanabe T, Izumi H, Kohno K, and Sasaguri $Y$. Overexpression of peroxiredoxin 4 protects against high-dose streptozotocin-induced diabetes by suppressing oxidative stress and cytokines in transgenic mice. Antioxid Redox Signal 13: 1477-1490, 2010.

6. Giguere P, Turcotte ME, Hamelin E, Parent A, Brisson J, Laroche G, Labrecque P, Dupuis G, and Parent JL. Peroxiredoxin- 4 interacts with and regulates the thromboxane $\mathrm{A}_{2}$ receptor. FEBS Lett 581: 3863-3868, 2007.

7. Guo X, Yamada S, Tanimoto A, Ding Y, Wang KY, Shimajiri S, Murata Y, Kimura S, Tasaki T, Nabeshima A, Watanabe T, Kohno K, and Sasaguri Y. Overexpression of peroxiredoxin 4 attenuates atherosclerosis in apolipoprotein E knockout mice. Antioxid Redox Signal 17: 1362-1375, 2012.

8. Havel PJ. Dietary fructose: implications for dysregulation of energy homeostasis and lipid/carbohydrate metabolism. Nutr Rev 63: 133-157, 2005.
9. Iuchi Y, Okada f, Tsunoda S, Kibe N, Shirasawa N, Ikawa M, Okabe M, Ikeda Y, and Fujii J. Peroxiredoxin 4 knockout results in elevated spermatogenic cell death via oxidative stress. Biochem J 419: 149-158, 2009.

10. Jin DY, Chae HZ, Rhee SG, and Jeang KT. Regulatory role for a novel human thioredoxin peroxidase in NF- $\kappa$ B activation. J Biol Chem 272: 30952-30961, 1997.

11. Kang SW, Rhee SG, Chang TS, Jeong W, and Choi MH. 2Cys peroxiredoxin function in intracellular signal transduction: therapeutic implications. Trends Mol Med 11: 571-578, 2005.

12. Kawasaki T, Igarashi $K$, Koeda T, Sugimoto $K$, Nakagawa $\mathrm{K}$, Hayashi S, Yamaji R, Inui H, Fukusato T, and Yamanouchi T. Rats fed fructose-enriched diets have characteristics of nonalcoholic hepatic steatosis. J Nutr 139: 20672071, 2009.

13. Kelley GL, Allan G, and Azhar S. High dietary fructose induces a hepatic stress response resulting in cholesterol and lipid dysregulation. Endocrinology 145: 548-555, 2004.

14. Kim $\mathrm{CH}$ and Younossi ZM. Nonalcoholic fatty liver disease: a manifestation of the metabolic syndrome. Cleve Clin J Med 75: 721-728, 2008.

15. Kita Y, Takamura T, Misu H, Ota T, Kurita S, Takeshita Y, Uno M, Matsuzawa-Nagata N, Kato K, Ando H, Fujimura A, Hayashi K, Kimura T, Ni Y, Otoda T, Miyamoto K, Zen Y, Nakanuma Y, and Kaneko S. Metformin prevents and reverses inflammation in a non-diabetic mouse model of nonalcoholic steatohepatitis. PLoS One 7: e43056, 2012.

16. Ludwig DS, Peterson KE, and Gortmaker SL. Relation between consumption of sugar-sweetened drinks and childhood obesity: a prospective, observational analysis. Lancet 357: 505-508, 2005.

17. Luo J, Quan J, Tsai J, Hobensack CK, Sullivan C, Hector R, and Reaven GM. Nongenetic mouse models of non-insulindependent diabetes mellitus. Metabolism 47: 663-668, 1998.

18. Matsumoto A, Okado A, Fujii T, J Fujii J, Egashira M, Niikawa N, and Taniguchi N. Cloning of the peroxiredoxin gene family in rats and characterization of the fourth member. FEBS Lett 443: 246-250, 1999.

19. Matteoni CA, Younossi ZM, Gramlich T, Boparai N, Liu YC, and McCullough AJ. Nonalcoholic fatty liver disease: a spectrum of clinical and pathological severity. Gastroenterology 116: 1413-1419, 1999.

20. Meier JL and Stinski MF. Regulation of human cytomegalovirus immediate-early gene expression. Intervirology 39: 331-342, 1996.

21. Mellor K, Ritchie RH, Meredith G, Woodman OL, Morris MJ and Delbridge LM. High-fructose diet elevates myocardial superoxide generation in mice in the absence of cardiac hypertrophy. Nutrition 26: 842-848, 2010.

22. Montonen J, Järvinen $R$, Knekt $P$, Heliövaara $M$, and Reunanen A. Consumption of sweetened beverages and intakes of fructose and glucose predict type 2 diabetes occurrence. J Nutr 137: 1447-1454, 2007.

23. Neuschwander-Tetri BA. Nonalcoholic steatohepatitis and the metabolic syndrome. Am J Med Sic 330: 326-335, 2005.

24. Rhee SG, Chae HZ, and Kim K. Peroxiredoxins: a historical overview and speculative preview of novel mechanisms and emerging concepts in cell signaling. Free Radic Biol Med 38: 1543-1552, 2005.

25. Syn WK, Choi SS, and Diehl AM. Apoptosis and cytokines in non-alcoholic steatohepatitis. Clin Liver Dis 13: 565-580, 2009. 
26. Tappy L, Lê KA, Tran C, and Paquot N. Fructose and metabolic diseases: new findings, new questions. Nutrition 26: 1044-1049, 2010.

27. Wang KY, Tanimoto A, Yamada S, Guo X, Ding Y, Watanabe T, Watanabe T, Kohno K, Hirano $K$, Tsukada $H$, and Sasaguri Y. Histamine regulation in glucose and lipid metabolism via histamine receptors: model for nonalcoholic steatohepatitis in mice. Am J Pathol 177: 713-723, 2010.

28. Wei Y, Wang D, and Pagliassotti MJ. Fructose selectively modulates c-jun N-terminal kinase activity and insulin signaling in rat primary hepatocytes. J Nutr 135: 1642-1646, 2005.

29. Yamada S, Ding Y, and Sasaguri Y. Peroxiredoxin 4: critical roles in inflammatory diseases. J UOEH 34: 27-39, 2012.

30. Yamaguchi K, Yang L, McCall S, Huang J, Yu XX, Pandey SK, Bhanot S, Monia BP, Li YX, and Diehl AM. Inhibiting triglyceride synthesis improves hepatic steatosis but exacerbates liver damage and fibrosis in obese mice with nonalcoholic steatohepatitis. Hepatology 45: 1366-1374, 2007.

31. Yamauchi T, Kamon J, Waki H, Terauchi Y, Kubota N, Hara K, Mori Y, Ide T, Murakami K, Tsuboyama-Kasaoka N, Ezaki O, Akanuma Y, Gavrilova O, Vinson C, Reitman ML, Kagechika H, Shudo K, Yoda M, Nakano Y, Tobe K, Nagai R, Kimura S, Tomita M, Froguel P, and Kadowaki T. The fatderived hormone adiponectin reverses insulin resistance associated with both lipoatrophy and obesity. Nat Med 7: 941-946, 2001.

Address correspondence to:
Dr. Sohsuke Yamada
Department of Pathology and Cell Biology
School of Medicine
University of Occupational and Environmental Health
Iseigaoka, Yahatanishi-ku
Kitakyushu 807-8555
Japan

E-mail: sousuke@med.uoeh-u.ac.jp

Date of first submission to ARS Central, September 10, 2012; date of final revised submission, March 10, 2013; date of acceptance, March 11, 2013.

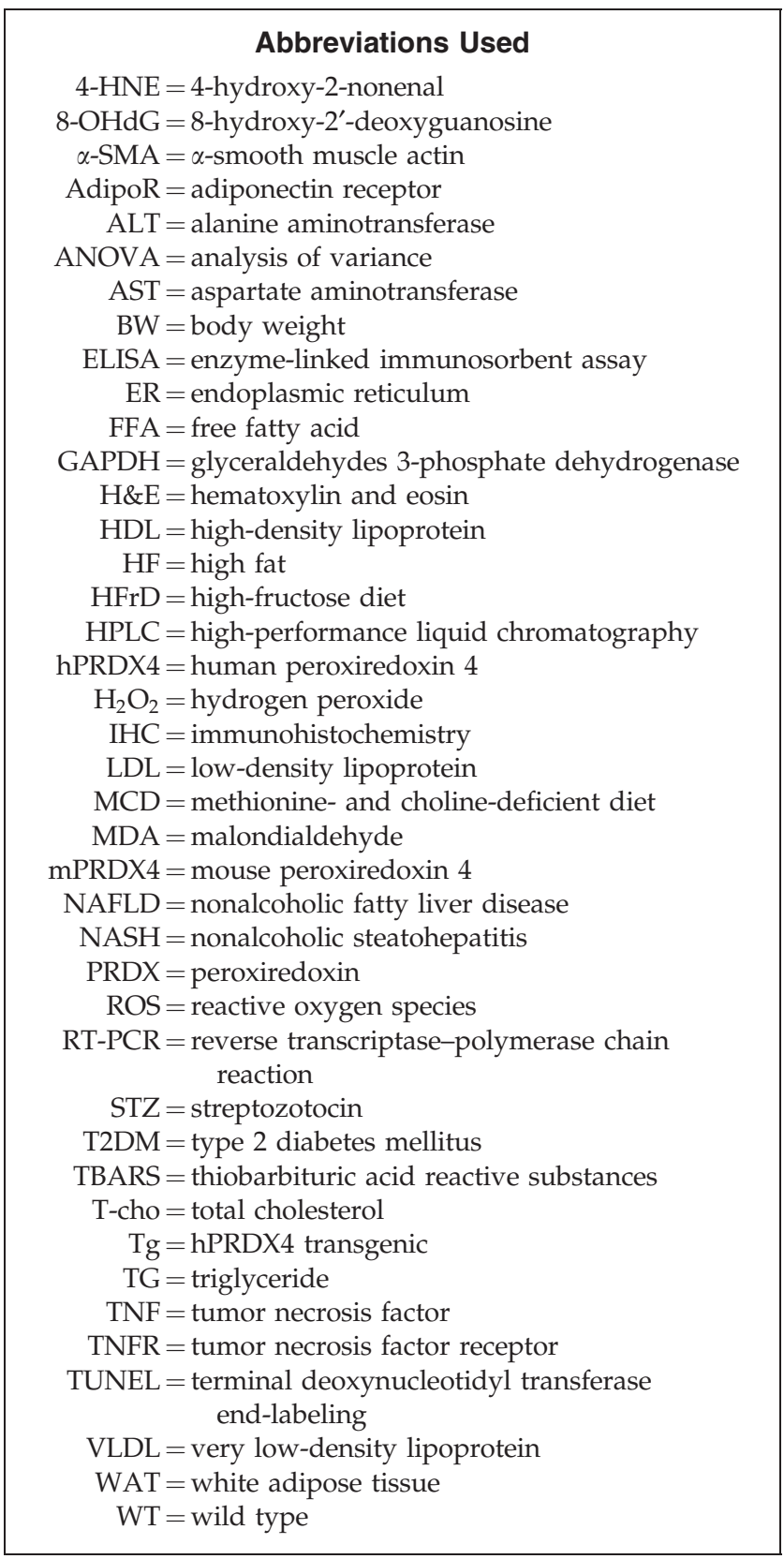

